\title{
Tests of the standard model in neutron beta decay with polarized electrons and unpolarized neutrons and protons
}

\author{
A. N. Ivanov, ${ }^{1, *}$ R. Höllwieser, ${ }^{1,2 \dagger}$ N. I. Troitskaya, ${ }^{1, \$}$ M. Wellenzohn, ${ }^{1,3,}$ and Ya. A. Berdnikov ${ }^{4, \|}$ \\ ${ }^{1}$ Atominstitut, Technische Universität Wien, Stadionallee 2, A-1020 Wien, Austria \\ ${ }^{2}$ Department of Physics, Bergische Universität Wuppertal, Gaussstr. 20, D-42119 Wuppertal, Germany \\ ${ }^{3}$ FH Campus Wien, University of Applied Sciences, Favoritenstraße 226, 1100 Wien, Austria \\ ${ }^{4}$ Peter the Great St. Petersburg Polytechnic University, \\ Polytechnicheskaya 29, 195251, Russian Federation
}

(Received 12 November 2018; published 20 March 2019)

\begin{abstract}
We calculate the correlation coefficients of the electron-energy and electron-antineutrino angular distribution of the neutron $\beta^{-}$-decay with polarized electron and unpolarised neutron and proton. The calculation is carried out within the standard model (SM) with the contributions, caused by the weak magnetism, proton recoil and radiative corrections of order of $10^{-3}$, Wilkinson's corrections of order $10^{-5}$ [Wilkinson, Nucl. Phys. A377, 474 (1982) and Ivanov et al., Phys. Rev. C 95, 055502 (2017)] and the contributions of interactions beyond the SM. The obtained results can be used for the analysis of experimental data on searches of interactions beyond the SM at the level of $10^{-4}$ [Abele, Hyperfine Interact. 237, 155 (2016)]. The contributions of $G$-odd correlations are calculated and found at the level of $10^{-5}$ in agreement with the results obtained by Gardner and Plaster [Phys. Rev. C 87, 065504 (2013)] and Ivanov et al. [Phys. Rev. C 98, 035503 (2018)].
\end{abstract}

DOI: 10.1103/PhysRevD.99.053004

\section{INTRODUCTION}

In Refs. [1-3] we have calculated the neutron lifetime and correlation coefficients of the electron-energy and angular distributions of the neutron $\beta^{-}$-decay with polarized neutron and unpolarized electron and proton, and polarized neutron and electron and an unpolarized proton, respectively. The neutron lifetime and correlation coefficients are calculated at the level of $10^{-3}$ of contributions of the weak magnetism and proton recoil of order $O\left(E_{e} / M\right)$, where $E_{e}$ is the electron energy and $M$ is an averaged nucleon mass, and radiative corrections of order $O(\alpha / \pi)$, where $\alpha$ is the fine-structure constant [4]. The radiative corrections of order $O(\alpha / \pi)$ to the neutron lifetime and correlation coefficients of the neutron $\beta^{-}$-decay with polarized neutron and unpolarized electron and proton have been calculated by Sirlin [5] and Shann [6] (for details of these calculations we relegate a reader to [7] and [1]). In turn, the radiative corrections of

\footnotetext{
*ivanov@kph.tuwien.ac.at

roman.hoellwieser@gmail.com

\#natroitskaya@yandex.ru

§max.wellenzohn@gmail.com

berdnikov@spbstu.ru
}

Published by the American Physical Society under the terms of the Creative Commons Attribution 4.0 International license. Further distribution of this work must maintain attribution to the author(s) and the published article's title, journal citation, and DOI. Funded by SCOAP ${ }^{3}$. order $O(\alpha / \pi)$ to the correlation coefficients of the neutron $\beta^{-}$-decay with polarized neutron and electron, and unpolarized proton have been calculated in [2]. Then, in [1,3] we have taken into account the contributions of interactions beyond the standard model (SM) to the neutron $\beta^{-}$-decay with polarized neutron and unpolarized electron and proton, and polarized neutron and electron, and unpolarized proton, respectively.

This paper is addressed to the calculation of the correlation coefficients of the electron-energy and electronantineutrino angular distribution of the neutron $\beta^{-}$-decay with polarized electron and unpolarized neutron and proton. We calculate a complete set of corrections of order $10^{-3}$ defined by the corrections of order $O\left(E_{e} / M\right)$, caused by the weak magnetism and proton recoil and calculated to nextto-leading order in the large nucleon mass expansion, and radiative corrections of order $O(\alpha / \pi)$, calculated to leading order in the large nucleon mass expansion. We discuss also Wilkinson's corrections of order $10^{-5}$ [8], which have been adapted to the neutron $\beta^{-}$-decay with polarized neutron and electron and unpolarized proton in Ref. [2]. In addition we take into account the contributions of interactions beyond the SM [9-20] (see also [1,3]) including the contributions of the second class currents (or the $G$-odd correlations) $[19,20]$ ) (see also [3]).

The paper is organized as follows. In Sec. II we write down the general expression for the electron-energy and electron-antineutrino angular distribution of the neutron $\beta^{-}$-decay with polarized electron and unpolarized neutron 
and proton. In Sec. III we discuss the renormalization procedure of the amplitude of the neutron $\beta^{-}$-decay, caused by the effective $V-A$ weak interaction and radiative corrections, calculated to order $O(\alpha / \pi)$ in the one-photon exchange approximation. In Sec. IV we calculate the renormalized electron-energy and electron-antineutrino angular distribution to order $O\left(E_{e} / M\right)$ and $O(\alpha / \pi)$, caused by the weak magnetism, proton recoil, and radiative corrections, dependent on the infrared cutoff $\mu$ and obtained within the finite-photon mass regularization $[1,5]$. In Sec. V using the Dirac wave function of the decay electron, distorted in the Coulomb field of the decay proton, we calculate the correlation coefficient $L\left(E_{e}\right)$, responsible for time reversal violation. In Sec. VI we write down the observable electron-energy and electron-antineutrino angular distribution, calculated in the SM to order $10^{-3}$, caused by the weak magnetism and proton recoil of order $O\left(E_{e} / M\right)$ and radiative corrections of order $O(\alpha / \pi)$. We show that the radiative corrections to the correlation coefficients $H\left(E_{e}\right)$ and $K\left(E_{e}\right)$ are defined by the functions $(\alpha / \pi) h_{n}^{(3)}\left(E_{e}\right)$ and $(\alpha / \pi) h_{n}^{(4)}\left(E_{e}\right)$, calculated for the first time in the present paper. The radiative corrections $(\alpha / \pi) h_{n}^{(3)}\left(E_{e}\right)$ and $(\alpha / \pi) h_{n}^{(4)}\left(E_{e}\right)$ are calculated in the Appendix and plotted in Fig. 3. In Sec. VII we adduce the analytical expressions for the correlation coefficients $a\left(E_{e}\right), G\left(E_{e}\right), H\left(E_{e}\right), K_{e}\left(E_{e}\right)$ and $L\left(E_{e}\right)$, calculated in the $\mathrm{SM}$ to order $10^{-3}$, caused by the weak magnetism, proton recoil, and radiative corrections. The obtained results can be used for the analysis of the experimental data on the neutron $\beta^{-}$-decay with polarized electron and unpolarized neutron and proton. In Sec. VIII we discuss Wilkinson's corrections of order $10^{-5}$, which have not been taken into account for the calculation of the correlation coefficients in Sec. VII. They are caused by (i) the proton recoil in the Coulomb electron-proton final-state interaction, (ii) the finite proton radius, (iii) the proton-lepton convolution, and (iv) the higher-order outer radiative corrections [8]. We calculate the contributions to the correlation coefficients, induced by the change of the Fermi function caused by the proton recoil in the electron-proton final-state Coulomb interaction. We plot these corrections in the electron-energy region $0.761 \mathrm{MeV} \leq E_{e} \leq 0.966 \mathrm{MeV}$ in Fig. 4. We point out that Wilkinson's corrections of order $10^{-5}$, caused by (ii) the finite proton radius, (iii) the proton-lepton convolution and (iv) the higher-order outer radiative corrections and calculated in [2], retain fully their shapes and values for the correlation coefficients analysed in the present paper. In Secs. IX and $X$ we calculate the contributions to the correlation coefficients, caused by interactions beyond the SM [9-20] (see also [1,3]), and give the correlation coefficients in the form suitable for the analysis of experimental data on searches of contributions of interactions beyond the SM [21] (see also [1,3]). In Sec. XI we discuss the obtained results and perspectives of the theoretical background to order $10^{-5}$, which goes beyond the scope of Wilkinson's corrections of order $10^{-5}$ $[2,22]$. In the Appendix we calculate the electron-energy and electron-antineutrino angular distribution of the neutron radiative $\beta^{-}$-decay with polarized electron and unpolarized neutron and proton. We use these results for a cancellation of the infrared divergences in the electronenergy and electron-antineutrino angular distribution of the neutron $\beta^{-}$-decay with polarized electron and unpolarized neutron and proton. The results, obtained in the Appendix can be also used for the experimental analysis of the neutron radiative $\beta^{-}$-decay with polarized electron and unpolarized neutron and proton.

\section{ELECTRON-ENERGY AND ELECTRON- ANTINEUTRINO ANGULAR DISTRIBUTION}

The electron-energy and electron-antineutrino angular distribution of the neutron $\beta^{-}$-decay with polarized electron and unpolarized neutron and proton can be written in the following form $[11,14]$

$$
\begin{aligned}
\frac{d^{5} \lambda_{n}\left(E_{e}, \vec{k}_{e}, \vec{\xi}_{e}, \vec{k}_{\nu}\right)}{d E_{e} d \Omega_{e} d \Omega_{\nu}}= & \left(1+3 \lambda^{2}\right) \frac{G_{F}^{2}\left|V_{u d}\right|^{2}}{32 \pi^{5}}\left(E_{0}-E_{e}\right)^{2} \sqrt{E_{e}^{2}-m_{e}^{2}} E_{e} F\left(E_{e}, Z=1\right) \zeta\left(E_{e}\right)\left\{1+a\left(E_{e}\right) \frac{\vec{k}_{e} \cdot \vec{k}_{\nu}}{E_{e} E_{\nu}}+G\left(E_{e}\right) \frac{\vec{\xi}_{e} \cdot \vec{k}_{e}}{E_{e}}\right. \\
& \left.+H\left(E_{e}\right) \frac{\vec{\xi}_{e} \cdot \vec{k}_{\nu}}{E_{\nu}}+K_{e}\left(E_{e}\right) \frac{\left(\vec{\xi}_{e} \cdot \vec{k}_{e}\right)\left(\vec{k}_{e} \cdot \vec{k}_{\nu}\right)}{\left(E_{e}+m_{e}\right) E_{e} E_{\nu}}+L\left(E_{e}\right) \frac{\vec{\xi}_{e} \cdot\left(\vec{k}_{e} \times \vec{k}_{\nu}\right)}{E_{e} E_{\nu}}+\cdots\right\} .
\end{aligned}
$$

where $d \Omega_{e}$ and $d \Omega_{\nu}$ are infinitesimal solid angles of the electron and antineutrino 3-momenta, $\lambda=-1.2750(9)$ is the axial coupling [23] (see also [24-26] and [1-3]), $G_{F}=1.1664 \times 10^{-11} \mathrm{MeV}^{-2}$ is the Fermi weak coupling constant, $V_{u d}=0.97417$ (21) is the Cabibbo-Kobayashi-Maskawa matrix element [4], extracted from the $0^{+} \rightarrow 0^{+}$transitions, $E_{0}=\left(m_{n}^{2}-m_{p}^{2}+m_{e}^{2}\right) / 2 m_{n}=1.2926 \mathrm{MeV}$ is the end-point energy of the electron spectrum, calculated for the neutron $m_{n}=939.5654 \mathrm{MeV}$, proton $m_{p}=938.2721 \mathrm{MeV}$, and electron $m_{e}=0.5110 \mathrm{MeV}$ masses [4], $\vec{\xi}_{e}$ is a unit polarization vector of the electron, and $F\left(E_{e}, Z=1\right)$ is the relativistic Fermi function used in [1-3] and equal to [27-29]

$$
F\left(E_{e}, Z=1\right)=\left(1+\frac{1}{2} \gamma\right) \frac{4\left(2 r_{p} m_{e} \beta\right)^{2 \gamma}}{\Gamma^{2}(3+2 \gamma)} \frac{e^{\pi \alpha / \beta}}{\left(1-\beta^{2}\right)^{\gamma}}\left|\Gamma\left(1+\gamma+i \frac{\alpha}{\beta}\right)\right|^{2}
$$


where $\beta=k_{e} / E_{e}=\sqrt{E_{e}^{2}-m_{e}^{2}} / E_{e}$ is the electron velocity, $\gamma=\sqrt{1-\alpha^{2}}-1, r_{p}$ is the electric radius of the proton. In the numerical calculations we will use $r_{p}=0.841 \mathrm{fm}$ [30].

The function $\zeta\left(E_{e}\right)$ and the correlation coefficients $a\left(E_{e}\right)$ and $G\left(E_{e}\right)$ have been calculated in [1-3]. They are defined by the contributions of order $10^{-3}$ of the SM interactions, Wilkinson's corrections of order $10^{-5}$ and interactions beyond the SM (see [1-3] and [31]). In this paper we calculate the correlation coefficients $H\left(E_{e}\right), K_{e}\left(E_{e}\right)$ and $L\left(E_{e}\right)$, where the correlation coefficient $L\left(E_{e}\right)$ is responsible for violation of invariance under transformation of time reversal. We calculate (i) a complete set of corrections of order $10^{-3}$, caused by the weak magnetism and proton recoil of order $O\left(E_{e} / M\right)$ and radiative corrections of order $O(\alpha / \pi)$, (ii) Wilkinson's corrections of order $10^{-5}$ [8] (see also [1,2]), (iii) contributions of interactions beyond the SM [11-14] (see also [1,3]), and (iv) second class contributions or $G$-odd correlations $[19,20]$ ) (see also [3]).

\section{EFFECTIVE LOW-ENERGY INTERACTIONS, DEFINING AMPLITUDE OF NEUTRON $\beta^{-}$-DECAY TO ORDER $10^{-3}$ IN THE SM}

In the SM of electroweak interactions the neutron $\beta^{-}$-decays, defined in the one-loop approximation with one-virtual-photon exchanges, are described by the following interactions

$$
\mathcal{L}_{\text {int }}(x)=\mathcal{L}_{\mathrm{W}}(x)+\mathcal{L}_{\mathrm{em}}(x) .
$$

Here $\mathcal{L}_{\mathrm{W}}(x)$ is the effective Lagrangian of low-energy $V-A$ interactions with a real axial coupling constant $\lambda=-1.2750(9)[23]$ (see also [1,2])

$$
\begin{aligned}
\mathcal{L}_{\mathrm{W}}(x)= & -\frac{G_{0 F}}{\sqrt{2}} V_{u d}\left\{\left[\bar{\psi}_{0 p}(x) \gamma_{\mu}\left(1+\lambda \gamma^{5}\right) \psi_{0 n}(x)\right]\right. \\
& \left.+\frac{\kappa}{2 M} \partial^{\nu}\left[\bar{\psi}_{0 p}(x) \sigma_{\mu \nu} \psi_{0 n}(x)\right]\right\} \\
& \times\left[\bar{\psi}_{0 e}(x) \gamma^{\mu}\left(1-\gamma^{5}\right) \psi_{0 \nu}(x)\right],
\end{aligned}
$$

where $\psi_{0 p}(x), \psi_{0 n}(x), \psi_{0 e}(x)$, and $\psi_{0 \nu}(x)$ are bare field operators of the proton, neutron, electron, and antineutrino, respectively, $G_{0 F}$ is a bare Fermi weak coupling constant, and $\gamma^{\mu}=\left(\gamma^{0}, \vec{\gamma}\right)$ and $\gamma^{5}$ are the Dirac matrices [32]; $\kappa=\kappa_{p}-\kappa_{n}=3.7058$ is the isovector anomalous magnetic moment of the nucleon, defined by the anomalous magnetic moments of the proton $\kappa_{p}=1.7928$ and the neutron $\kappa_{n}=-1.9130$ and measured in nuclear magneton [4], and $M=\left(m_{n}+m_{p}\right) / 2$ is the average nucleon mass.

For the calculation of the radiative corrections to order $O(\alpha / \pi)$ the Lagrangian of the electromagnetic interaction $\mathcal{L}_{\mathrm{em}}(x)$ we take in the following form [22]

$$
\begin{aligned}
\mathcal{L}_{\mathrm{em}}(x)= & -\frac{1}{4} F_{\mu \nu}^{(0)}(x) F^{(0) \mu \nu}(x)-\frac{1}{2 \xi_{0}}\left(\partial_{\mu} A^{(0) \mu}(x)\right)^{2} \\
& +\bar{\psi}_{0 e}(x)\left(i \gamma^{\mu} \partial_{\mu}-m_{0 e}\right) \psi_{0 e}(x) \\
& -\left(-e_{0}\right) \bar{\psi}_{0 e}(x) \gamma^{\mu} \psi_{0 e}(x) A_{\mu}^{(0)}(x) \\
& +\bar{\psi}_{0 p}(x)\left(i \gamma^{\mu} \partial_{\mu}-m_{0 p}\right) \psi_{0 p}(x) \\
& -\left(+e_{0}\right) \bar{\psi}_{0 p}(x) \gamma^{\mu} \psi_{0 p}(x) A_{\mu}^{(0)}(x)
\end{aligned}
$$

where $F_{\mu \nu}^{(0)}(x)=\partial_{\mu} A_{\nu}^{(0)}(x)-\partial_{\nu} A_{\mu}^{(0)}(x)$ is the electromagnetic field strength tensor of the bare (unrenormalized) electromagnetic field operator $A_{\mu}^{(0)}(x) ; \psi_{0 e}(x)$ and $\psi_{0 p}(x)$ are bare operators of the electron and proton fields with bare masses $m_{0 e}$ and $m_{0 p}$, respectively; $-e_{0}$ and $+e_{0}$ are bare electric charges of the electron and proton, respectively. Then, $\xi_{0}$ is a bare gauge parameter. After the calculation of the one-loop corrections of order $O(\alpha / \pi)$ a transition to the renormalized field operators, masses and electric charges is defined by the Lagrangian

$$
\begin{aligned}
\mathcal{L}_{\mathrm{em}}(x)= & -\frac{1}{4} F_{\mu \nu}(x) F^{\mu \nu}(x)-\frac{1}{2 \xi}\left(\partial_{\mu} A^{\mu}(x)\right)^{2} \\
& +\bar{\psi}_{e}(x)\left(i \gamma^{\mu} \partial_{\mu}-m_{e}\right) \psi_{e}(x) \\
& -(-e) \bar{\psi}_{e}(x) \gamma^{\mu} \psi_{e}(x) A_{\mu}(x) \\
& +\bar{\psi}_{p}(x)\left(i \gamma^{\mu} \partial_{\mu}-m_{p}\right) \psi_{p}(x) \\
& -(+e) \bar{\psi}_{p}(x) \gamma^{\mu} \psi_{p}(x) A_{\mu}(x)+\delta \mathcal{L}_{\mathrm{em}}(x),
\end{aligned}
$$

where $A_{\mu}(x), \psi_{e}(x)$ and $\psi_{p}(x)$ are the renormalized operators of the electromagnetic, electron and proton fields, respectively; $m_{e}$ and $m_{p}$ are the renormalized masses of the electron and proton; $e$ is the renormalized electric charge; and $\xi$ is the renormalized gauge parameter. The Lagrangian $\delta \mathcal{L}_{\mathrm{em}}(x)$ contains a complete set of the counterterms [33],

$$
\begin{aligned}
\delta \mathcal{L}_{\mathrm{em}}(x)= & -\frac{1}{4}\left(Z_{3}-1\right) F_{\mu \nu}(x) F^{\mu \nu}(x)-\frac{Z_{3}-1}{Z_{\xi}} \frac{1}{2 \xi}\left(\partial_{\mu} A^{\mu}(x)\right)^{2}+\left(Z_{2}^{(e)}-1\right) \bar{\psi}_{e}(x)\left(i \gamma^{\mu} \partial_{\mu}-m_{e}\right) \psi_{e}(x) \\
& -\left(Z_{1}^{(e)}-1\right)(-e) \bar{\psi}_{e}(x) \gamma^{\mu} \psi_{e}(x) A_{\mu}(x)-Z_{2}^{(e)} \delta m_{e} \bar{\psi}_{e}(x) \psi_{e}(x)+\left(Z_{2}^{(p)}-1\right) \bar{\psi}_{p}(x)\left(i \gamma^{\mu} \partial_{\mu}-m_{p}\right) \psi_{p}(x) \\
& -\left(Z_{1}^{(p)}-1\right)(+e) \bar{\psi}_{p}(x) \gamma^{\mu} \psi_{p}(x) A_{\mu}(x)-Z_{2}^{(p)} \delta m_{p} \bar{\psi}_{p}(x) \psi_{p}(x),
\end{aligned}
$$


where $Z_{3}, Z_{2}^{(e)}, Z_{1}^{(e)}, Z_{2}^{(p)}, Z_{1}^{(p)}, \delta m_{e}$, and $\delta m_{p}$ are the counterterms. Here $Z_{3}$ is the renormalization constant of the electromagnetic field operator $A_{\mu}, Z_{2}^{(e)}$ and $Z_{1}^{(e)}$ are the renormalization constants of the electron field operator $\psi_{e}$ and the electron-electron-photon $\left(e^{-} e^{-} \gamma\right)$ vertex, respectively; $Z_{2}^{(p)}$ and $Z_{1}^{(p)}$ are the renormalization constants of the proton field operator $\psi_{p}$ and the proton-proton-photon $(p p \gamma)$ vertex, respectively. Then, $(-e)$ and $(+e), m_{e}$ and $m_{p}$ and $\delta m_{e}$ and $\delta m_{p}$ are the renormalized electric charges and masses and the mass-counterterms of the electron and proton, respectively. Rescaling the field operators $[33,34]$

$$
\sqrt{Z_{3}} A_{\mu}(x)=A_{\mu}^{(0)}(x), \quad \sqrt{Z_{2}^{(e)}} \psi_{e}(x)=\psi_{0 e}(x), \quad \sqrt{Z_{2}^{(p)}} \psi_{p}(x)=\psi_{0 p}(x)
$$

and denoting $m_{e}+\delta m_{e}=m_{0 e}, m_{p}+\delta m_{p}=m_{0 p}$, and $Z_{\xi} \xi=\xi_{0}$ we arrive at the Lagrangian

$$
\begin{aligned}
\mathcal{L}_{\mathrm{em}}(x)= & -\frac{1}{4} F_{\mu \nu}^{(0)}(x) F^{(0) \mu \nu}(x)-\frac{1}{2 \xi_{0}}\left(\partial_{\mu} A^{(0) \mu}(x)\right)^{2}+\bar{\psi}_{0 e}(x)\left(i \gamma^{\mu} \partial_{\mu}-m_{0 e}\right) \psi_{0 e}(x) \\
& -(-e) Z_{1}^{(e)}\left(Z_{2}^{(e)}\right)^{-1} Z_{3}^{-1 / 2} \bar{\psi}_{0 e}(x) \gamma^{\mu} \psi_{0 e}(x) A_{\mu}^{(0)}(x)+\bar{\psi}_{0 p}(x)\left(i \gamma^{\mu} \partial_{\mu}-m_{0 p}\right) \psi_{0 p}(x) \\
& -(+e) Z_{1}^{(p)}\left(Z_{2}^{(p)}\right)^{-1} Z_{3}^{-1 / 2} \bar{\psi}_{0 p}(x) \gamma^{\mu} \psi_{0 p}(x) A_{\mu}^{(0)}(x) .
\end{aligned}
$$

Because of the Ward identities $Z_{1}^{(e)}=Z_{2}^{(e)}$ and $Z_{1}^{(p)}=Z_{2}^{(p)}$ [32-34], we may replace $(-e) Z_{3}^{-1 / 2}=-e_{0}$ and $(+e) Z_{3}^{-1 / 2}=+e_{0}$. This brings Eq. (9) to the form of Eq. (5). We would like to emphasize that to order $O(\alpha / \pi)$ the renormalization constant $Z_{3}$ is equal to unity because of the absent of closed fermion loops [32-34], i.e., $Z_{3}=1$. This means that in such an approximation the bare electric charge $e_{0}$ coincides with the renormalized electric charge $e$, i.e., $e_{0}=e$. After the rescaling of the proton and electron field operators Eq. (8) the Lagrangian of $V-A$ weak interactions Eq. (4) takes the form

$$
\mathcal{L}_{\mathrm{W}}(x)=-\frac{G_{F}}{\sqrt{2}} V_{u d}\left\{\left[\bar{\psi}_{p}(x) \gamma_{\mu}\left(1+\lambda \gamma^{5}\right) \psi_{n}(x)\right]+\frac{\kappa}{2 M} \partial^{\nu}\left[\bar{\psi}_{p}(x) \sigma_{\mu \nu} \psi_{n}(x)\right]\right\}\left[\bar{\psi}_{e}(x) \gamma^{\mu}\left(1-\gamma^{5}\right) \psi_{\nu}(x)\right],
$$

where $G_{F}=\sqrt{Z_{2}^{(p)} Z_{2}^{(e)}} G_{0 F}$ is the Fermi weak coupling constant renormalized by electromagnetic interactions to order $O(\alpha / \pi)$. The bare neutron $\psi_{0 n}(x)$ and antineutrino $\psi_{0 \nu}(x)$ field operators are not renormalized by electromagnetic interactions and coincide with the field operators $\psi_{n}(x)$ and $\psi_{\nu}(x)$, respectively, i.e., $\psi_{0 n}(x)=\psi_{n}(x)$ and $\psi_{0 \nu}(x)=\psi_{\nu}(x)$.

\section{ELECTRON-ENERGY AND ELECTRON-ANTINEUTRINO ANGULAR DISTRIBUTION WITH RADIATIVE CORRECTIONS CAUSED BY ONE-VIRTUAL PHOTON EXCHANGES}

Using the results, obtained in [1], the renormalized amplitude of the neutron $\beta^{-}$-decay with contributions, caused by the weak magnetism and proton recoil, calculated to next-to-leading order $O\left(E_{e} / M\right)$ in the large nucleon mass expansion, and radiative corrections to order $O(\alpha / \pi)$, defined by the Feynman diagrams in Fig. 1 and calculated to leading order in the large nucleon mass expansion, takes the form (see Eq. (D-52) of Ref. [1])

$$
\begin{aligned}
M\left(n \rightarrow p e^{-} \bar{\nu}_{e}\right)= & -2 m_{n} \frac{G_{F}}{\sqrt{2}} V_{u d}\left\{\left(1+\frac{\alpha}{2 \pi} f_{\beta_{c}^{-}}\left(E_{e}, \mu\right)\right)\left[\varphi_{p}^{\dagger} \varphi_{n}\right]\left[\bar{u}_{e} \gamma^{0}\left(1-\gamma^{5}\right) v_{\bar{\nu}}\right]\right. \\
& -\tilde{\lambda}\left(1+\frac{\alpha}{2 \pi} f_{\beta_{c}^{-}}\left(E_{e}, \mu\right)\right)\left[\varphi_{p}^{\dagger} \vec{\sigma} \varphi_{n}\right] \cdot\left[\bar{u}_{e} \vec{\gamma}\left(1-\gamma^{5}\right) v_{\bar{\nu}}\right]-\frac{\alpha}{2 \pi} g_{F}\left(E_{e}\right)\left[\varphi_{p}^{\dagger} \varphi_{n}\right]\left[\bar{u}_{e}\left(1-\gamma^{5}\right) v_{\bar{\nu}}\right] \\
& +\frac{\alpha}{2 \pi} \tilde{\lambda}_{F}\left(E_{e}\right)\left[\varphi_{p}^{\dagger} \vec{\sigma} \varphi_{n}\right] \cdot\left[\bar{u}_{e} \gamma^{0} \vec{\gamma}\left(1-\gamma^{5}\right) v_{\bar{\nu}}\right]-\frac{m_{e}}{2 M}\left[\varphi_{p}^{\dagger} \varphi_{n}\right]\left[\bar{u}_{e}\left(1-\gamma^{5}\right) v_{\bar{\nu}}\right] \\
& \left.+\frac{\tilde{\lambda}}{2 M}\left[\varphi_{p}^{\dagger}\left(\vec{\sigma} \cdot \vec{k}_{p}\right) \varphi_{n}\right]\left[\bar{u}_{e} \gamma^{0}\left(1-\gamma^{5}\right) v_{\bar{\nu}}\right]-i \frac{\kappa+1}{2 M}\left[\varphi_{p}^{\dagger}\left(\vec{\sigma} \times \vec{k}_{p}\right) \varphi_{n}\right] \cdot\left[\bar{u}_{e} \vec{\gamma}\left(1-\gamma^{5}\right) v_{\bar{\nu}}\right]\right\},
\end{aligned}
$$

where $\varphi_{p}$ and $\varphi_{n}$ are Pauli spinorial wave functions of the proton and neutron, $u_{e}$ and $v_{\nu}$ are Dirac wave functions of the electron and electron antineutrino, $\vec{\sigma}$ are the Pauli $2 \times 2$ matrices, and $\tilde{\lambda}=\lambda\left(1-E_{0} / 2 M\right)$ and $\vec{k}_{p}=-\vec{k}_{e}-\vec{k}_{\nu}$ is the proton 3 -momentum in the rest frame of the neutron. The functions $f_{\beta_{c}^{-}}\left(E_{e}, \mu\right)$ and $g_{F}\left(E_{e}\right)$ are equal to [see Eq. (D-51)] 


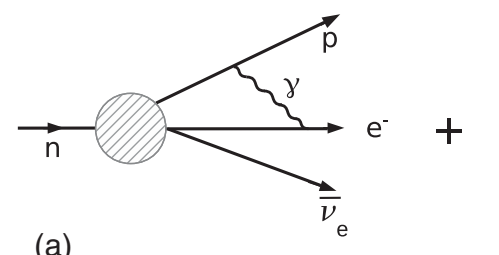

(a)

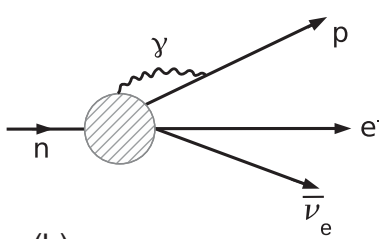

(b)

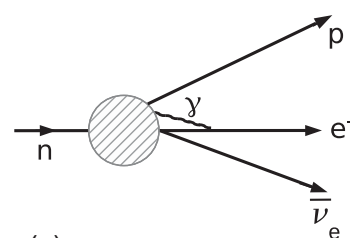

(c)

FIG. 1. The Feynman diagrams, defining the main contribution of the radiative corrections of order $O(\alpha / \pi)$, caused by one-virtual photon exchanges, to the neutron $\beta^{-}$-decay (see Sirlin [5]).

$$
\begin{aligned}
f_{\beta_{c}^{-}}\left(E_{e}, \mu\right)= & \frac{3}{2} \ell n\left(\frac{m_{p}}{m_{e}}\right)-\frac{11}{8}+2 \ell n\left(\frac{\mu}{m_{e}}\right)\left[\frac{1}{2 \beta} \ln \left(\frac{1+\beta}{1-\beta}\right)-1\right]-\frac{1}{\beta} \operatorname{Li}_{2}\left(\frac{2 \beta}{1+\beta}\right)-\frac{1}{4 \beta} \ln n^{2}\left(\frac{1+\beta}{1-\beta}\right) \\
& +\frac{1}{2 \beta} \ln \left(\frac{1+\beta}{1-\beta}\right)+C_{W Z}, \\
g_{F}\left(E_{e}\right)= & \frac{\sqrt{1-\beta^{2}}}{2 \beta} \ln \left(\frac{1+\beta}{1-\beta}\right),
\end{aligned}
$$

where $\mu$ is a photon mass, which should be taken in the limit $\mu \rightarrow 0$, and $\operatorname{Li}_{2}(x)$ is the Polylogarithmic function. A photon mass $\mu$ is used for Lorentz invariant regularization of infrared divergences of radiative corrections [5]. The constant $C_{W Z}$, defined by the contributions of the $W$-boson and $Z$-boson exchanges and the QCD corrections [35] (see also [36,37]), is equal to $C_{W Z}=10.249$ (see also discussion below Eq. (D-58) of Ref. [1]).

The squared absolute value of the matrix element Eq. (12), summed over polarizations of massive fermions, we calculate for polarized electron and unpolarized neutron and proton [2]. We get (see also Eq. (A-16) in Appendix A of Ref. [1])

$$
\begin{aligned}
& \sum_{\text {pol }} \frac{\left|M\left(n \rightarrow p e^{-} \nu_{e}\right)\right|^{2}}{8 m_{n}^{2} G_{F}^{2}\left|V_{u d}\right|^{2}} \\
& =\left(1+\frac{\alpha}{\pi} f_{\beta_{c}^{-}}\left(E_{e}, \mu\right)\right) \operatorname{tr}\left\{\left(\hat{k}_{e}+m_{e}\right)\left(1+\gamma^{5} \hat{\zeta}_{e}\right) \gamma^{0} \hat{k}_{\nu} \gamma^{0}\left(1-\gamma^{5}\right)\right\}-\frac{\alpha}{2 \pi} g_{F}\left(E_{e}\right) \operatorname{tr}\left\{\left(\hat{k}_{e}+m_{e}\right)\left(1+\gamma^{5} \hat{\zeta}_{e}\right) \gamma^{0} \hat{k}_{\nu}\left(1+\gamma^{5}\right)\right\} \\
& -\frac{\alpha}{2 \pi} g_{F}\left(E_{e}\right) \operatorname{tr}\left\{\left(\hat{k}_{e}+m_{e}\right)\left(1+\gamma^{5} \hat{\zeta}_{e}\right) \hat{k}_{\nu} \gamma^{0}\left(1-\gamma^{5}\right)\right\}+\tilde{\lambda}^{2}\left(1+\frac{\alpha}{\pi} f_{\beta_{c}^{-}}\left(E_{e}, \mu\right)\right) \delta^{i j} \operatorname{tr}\left\{\left(\hat{k}_{e}+m_{e}\right)\left(1+\gamma^{5} \hat{\zeta}_{e}\right) \gamma^{j} \hat{k}_{\nu} \gamma^{i}\left(1-\gamma^{5}\right)\right\} \\
& -\tilde{\lambda}^{2} \frac{\alpha}{2 \pi} g_{F}\left(E_{e}\right) \delta^{i j} \operatorname{tr}\left\{\left(\hat{k}_{e}+m_{e}\right)\left(1+\gamma^{5} \hat{\zeta}_{e}\right) \gamma^{0} \gamma^{i} \hat{k}_{\nu} \gamma^{j}\left(1-\gamma^{5}\right)\right\}+\tilde{\lambda}^{2} \frac{\alpha}{2 \pi} g_{F}\left(E_{e}\right) \delta^{i j} \operatorname{tr}\left\{\left(\hat{k}_{e}+m_{e}\right)\left(1+\gamma^{5} \hat{\zeta}_{e}\right) \gamma^{i} \hat{k}_{\nu} \gamma^{0} \gamma^{j}\left(1+\gamma^{5}\right)\right\} \\
& -\frac{m_{e}}{2 M} \operatorname{tr}\left\{\left(\hat{k}_{e}+m_{e}\right)\left(1+\gamma^{5} \hat{\zeta}_{e}\right) \gamma^{0} \hat{k}_{\nu}\left(1+\gamma^{5}\right)\right\}-\frac{m_{e}}{2 M} \operatorname{tr}\left\{\left(\hat{k}_{e}+m_{e}\right)\left(1+\gamma^{5} \hat{\zeta}_{e}\right) \hat{k}_{\nu} \gamma^{0}\left(1-\gamma^{5}\right)\right\} \\
& -\frac{\tilde{\lambda}^{2}}{2 M} \vec{k}_{p} \cdot \operatorname{tr}\left\{\left(\hat{k}_{e}+m_{e}\right)\left(1+\gamma^{5} \hat{\zeta}_{e}\right) \vec{\gamma} \hat{k}_{\nu} \gamma^{0}\left(1-\gamma^{5}\right)\right\}-\frac{\tilde{\lambda}^{2}}{2 M} \vec{k}_{p} \cdot \operatorname{tr}\left\{\left(\hat{k}_{e}+m_{e}\right)\left(1+\gamma^{5} \hat{\zeta}_{e}\right) \gamma^{0} \hat{k}_{\nu} \vec{\gamma}\left(1-\gamma^{5}\right)\right\} \\
& +i \tilde{\lambda} \frac{\kappa+1}{2 M} \varepsilon^{\ell j a} k_{p}^{a} \operatorname{tr}\left\{\left(\hat{k}_{e}+m_{e}\right)\left(1+\gamma^{5} \hat{\zeta}_{e}\right) \gamma^{\ell} \hat{k}_{\nu} \gamma^{j}\left(1-\gamma^{5}\right)\right\}-i \tilde{\lambda} \frac{\kappa+1}{2 M} \varepsilon^{\ell j a} k_{p}^{a} \operatorname{tr}\left\{\left(\hat{k}_{e}+m_{e}\right)\left(1+\gamma^{5} \hat{\zeta}_{e}\right) \gamma^{j} \hat{k}_{\nu} \gamma^{\ell}\left(1-\gamma^{5}\right)\right\},
\end{aligned}
$$

where $\zeta_{e}^{\mu}=\left(\zeta_{e}^{0}, \vec{\zeta}_{e}\right)$ is the 4-vector of an electron polarization defined by [2]

$$
\zeta_{e}^{\mu}=\left(\zeta_{e}^{0}, \vec{\zeta}_{e}\right)=\left(\frac{\vec{k}_{e} \cdot \vec{\xi}_{e}}{m_{e}}, \vec{\xi}_{e}+\frac{\vec{k}_{e}\left(\vec{k}_{e} \cdot \vec{\xi}_{e}\right)}{m_{e}\left(E_{e}+m_{e}\right)}\right)
$$

It obeys the constraints $\zeta_{e}^{2}=-1$ and $k_{e} \cdot \zeta_{e}=0$, where $\vec{\xi}_{e}$ is a unit vector of the electron polarization [32]. We would like to emphasize that in Eq. (13) following Sirlin [5] we have neglected the contributions of order $O\left(\alpha E_{e} / \pi M\right)$. Having calculated the traces over Dirac matrices we obtain 


$$
\begin{aligned}
\sum_{\mathrm{pol}} & \frac{\mid}{32 m_{n}^{2} G_{F}^{2}\left|V_{u d}\right|^{2} E_{e} E_{\nu}} \\
= & \left(1+\frac{\alpha}{\pi} f_{\beta_{c}^{-}}\left(E_{e}, \mu\right)\right)\left(1+\frac{\vec{k}_{e} \cdot \vec{k}_{\nu}}{E_{e} E_{\nu}}-\frac{\vec{\xi}_{e} \cdot \vec{k}_{e}}{E_{e}}-\frac{m_{e}}{E_{e}} \frac{\vec{\xi}_{e} \cdot \vec{k}_{\nu}}{E_{\nu}}-\frac{\left(\vec{\xi}_{e} \cdot \vec{k}_{e}\right)\left(\vec{k}_{e} \cdot \vec{k}_{\nu}\right)}{\left(E_{e}+m_{e}\right) E_{e} E_{\nu}}\right)-\frac{\alpha}{\pi} g_{F}\left(E_{e}\right)\left(\frac{m_{e}}{E_{e}}+\frac{\vec{k}_{e} \cdot \vec{k}_{\nu}}{E_{e} E_{\nu}}-\frac{\vec{\xi}_{e} \cdot \vec{k}_{\nu}}{E_{\nu}}\right. \\
& \left.-\frac{E_{e}}{m_{e}} \frac{\left(\vec{\xi}_{e} \cdot \vec{k}_{e}\right)\left(\vec{k}_{e} \cdot \vec{k}_{\nu}\right)}{\left(E_{e}+m_{e}\right) E_{e} E_{\nu}}\right)+\tilde{\lambda}^{2}\left(1+\frac{\alpha}{\pi} f_{\beta_{c}^{-}}\left(E_{e}, \mu\right)\right)\left(3-\frac{\vec{k}_{e} \cdot \vec{k}_{\nu}}{E_{e} E_{\nu}}-3 \frac{\vec{\xi}_{e} \cdot \vec{k}_{e}}{E_{e}}+\frac{m_{e} \vec{\xi}_{e} \cdot \vec{k}_{\nu}}{E_{e}}+\frac{\left(\vec{\xi}_{e} \cdot \vec{k}_{e}\right)\left(\vec{k}_{e} \cdot \vec{k}_{\nu}\right)}{\left(E_{e}+m_{e}\right) E_{e} E_{\nu}}\right) \\
& -\tilde{\lambda}^{2} \frac{\alpha}{\pi} g_{F}\left(E_{e}\right)\left(3 \frac{m_{e}}{E_{e}}+\frac{\vec{\xi}_{e} \cdot \vec{k}_{\nu}}{E_{\nu}}-\frac{\left(\vec{\xi}_{e} \cdot \vec{k}_{e}\right)\left(\vec{k}_{e} \cdot \vec{k}_{\nu}\right)}{\left(E_{e}+m_{e}\right) E_{e} E_{\nu}}\right)-\frac{m_{e}}{M}\left(\frac{m_{e}}{E_{e}}-\frac{\vec{\xi}_{e} \cdot \vec{k}_{\nu}}{E_{\nu}}+\frac{\left(\vec{\xi}_{e} \cdot \vec{k}_{e}\right)\left(\vec{k}_{e} \cdot \vec{k}_{\nu}\right)}{\left(E_{e}+m_{e}\right) E_{e} E_{\nu}}\right)+\frac{\tilde{\lambda}^{2}}{M}\left(\left(E_{0}-\frac{m_{e}^{2}}{E_{e}}\right)\right. \\
& \left.+E_{0} \frac{\vec{k}_{e} \cdot \vec{k}_{\nu}}{E_{e} E_{\nu}}-E_{0} \frac{\vec{\xi}_{e} \cdot \vec{k}_{e}}{E_{e}}-E_{\nu} \frac{m_{e}}{E_{e}} \frac{\vec{\xi}_{e} \cdot \vec{k}_{\nu}}{E_{\nu}}-\left(E_{0}+m_{e}\right) \frac{\left(\vec{\xi}_{e} \cdot \vec{k}_{e}\right)\left(\vec{k}_{e} \cdot \vec{k}_{\nu}\right)}{\left(E_{e}+m_{e}\right) E_{e} E_{\nu}}\right)+\tilde{\lambda}^{2} \frac{2(\kappa+1)}{M}\left(\left(E_{0}-2 E_{e}+\frac{m_{e}^{2}}{E_{e}}\right)\right. \\
& \left.+\left(2 E_{e}-E_{0}\right) \frac{\vec{k}_{e} \cdot \vec{k}_{\nu}}{E_{e} E_{\nu}}+\left(2 E_{e}-E_{0}\right) \frac{\vec{\xi}_{e} \cdot \vec{k}_{e}}{E_{e}}+\left(E_{0}-E_{e}\right) \frac{m_{e}}{E_{e}} \frac{\vec{\xi}_{e} \cdot \vec{k}_{\nu}}{E_{\nu}}+\left(E_{0}-m_{e}\right) \frac{\left(\vec{\xi}_{e} \cdot \vec{k}_{e}\right)\left(\vec{k}_{e} \cdot \vec{k}_{\nu}\right)}{\left(E_{e}+m_{e}\right) E_{e} E_{\nu}}\right),
\end{aligned}
$$

where we have used a relation $E_{e}+E_{\nu}=E_{0}$. Now we have to take into account the contribution of the phase-volume [1] and multiply Eq. (15) by the function

$$
\Phi_{\beta_{c}^{-}}\left(\vec{k}_{e}, \vec{k}_{\nu}\right)=1+\frac{3}{M}\left(E_{e}-\frac{\vec{k}_{e} \cdot \vec{k}_{\nu}}{E_{\nu}}\right) .
$$

This gives

$$
\begin{aligned}
\Phi_{\beta_{c}^{-}} & \left(\vec{k}_{e}, \vec{k}_{\nu}\right) \sum_{\mathrm{pol}} \frac{\left|M\left(n \rightarrow p e^{-} \nu_{e}\right)\right|^{2}}{32 m_{n}^{2} G_{F}^{2}\left|V_{u d}\right|^{2} E_{e} E_{\nu}} \\
= & \left(1+3 \lambda^{2}\right) \tilde{\zeta}\left(E_{e}\right)\left\{1+\tilde{a}\left(E_{e}\right) \frac{\vec{k}_{e} \cdot \vec{k}_{\nu}}{E_{e} E_{\nu}}+\tilde{G}\left(E_{e}\right) \frac{\vec{\xi}_{e} \cdot \vec{k}_{e}}{E_{e}}+\tilde{H}\left(E_{e}\right) \frac{\vec{\xi}_{e} \cdot \vec{k}_{\nu}}{E_{\nu}}+\tilde{K}_{e}\left(E_{e}\right) \frac{\left(\vec{\xi}_{e} \cdot \vec{k}_{e}\right)\left(\vec{k}_{e} \cdot \vec{k}_{\nu}\right)}{\left(E_{e}+m_{e}\right) E_{e} E_{\nu}}\right. \\
& -3 a_{0} \frac{E_{e}}{M}\left(\frac{\left(\vec{k}_{e} \cdot \vec{k}_{\nu}\right)^{2}}{E_{e}^{2} E_{\nu}^{2}}-\frac{1}{3} \frac{k_{e}^{2}}{E_{e}^{2}}\right)+3 a_{0} \frac{m_{e}}{M}\left(\frac{\left(\vec{\xi}_{e} \cdot \vec{k}_{\nu}\right)\left(\vec{k}_{e} \cdot \vec{k}_{\nu}\right)}{E_{e} E_{\nu}^{2}}-\frac{1}{3} \frac{\vec{\xi}_{e} \cdot \vec{k}_{e}}{E_{e}}\right) \\
& \left.+3 a_{0} \frac{1}{M}\left(\frac{\left(\vec{\xi}_{e} \cdot \vec{k}_{e}\right)\left(\vec{k}_{e} \cdot \vec{k}_{\nu}\right)^{2}}{\left(E_{e}+m_{e}\right) E_{e} E_{\nu}^{2}}-\frac{1}{3}\left(E_{e}-m_{e}\right) \frac{\vec{\xi}_{e} \cdot \vec{k}_{e}}{E_{e}}\right)\right\},
\end{aligned}
$$

where we have denoted $a_{0}=\left(1-\lambda^{2}\right) /\left(1+3 \lambda^{2}\right)$ and

$$
\begin{aligned}
\tilde{\zeta}\left(E_{e}\right)= & \left(1+\frac{\alpha}{\pi} f_{\beta_{c}^{-}}\left(E_{e}, \mu\right)-\frac{\alpha}{\pi} g_{F}\left(E_{e}\right) \frac{m_{e}}{E_{e}}\right)+\frac{1}{M} \frac{1}{1+3 \lambda^{2}}\left[-2 \lambda(\lambda-(\kappa+1)) E_{0}\right. \\
& \left.+\left(10 \lambda^{2}-4(\kappa+1) \lambda+2\right) E_{e}-2 \lambda(\lambda-(\kappa+1)) \frac{m_{e}^{2}}{E_{e}}\right], \\
\tilde{\zeta}\left(E_{e}\right) \tilde{a}\left(E_{e}\right)= & a_{0}\left(1+\frac{\alpha}{\pi} f_{\beta_{c}^{-}}\left(E_{e}, \mu\right)\right)+\frac{1}{M} \frac{1}{1+3 \lambda^{2}}\left[2 \lambda(\lambda-(\kappa+1)) E_{0}-4 \lambda(3 \lambda-(\kappa+1)) E_{e}\right], \\
\tilde{\zeta}\left(E_{e}\right) \tilde{G}\left(E_{e}\right)= & -\left(1+\frac{\alpha}{\pi} f_{\beta_{c}^{-}}\left(E_{e}, \mu\right)\right)+\frac{1}{M} \frac{1}{1+3 \lambda^{2}}\left[2 \lambda(\lambda-(\kappa+1)) E_{0}-\left(10 \lambda^{2}-4(\kappa+1) \lambda+2\right) E_{e}\right] \\
\tilde{\zeta}\left(E_{e}\right) \tilde{H}\left(E_{e}\right)= & \frac{m_{e}}{E_{e}}\left\{-a_{0}\left(1+\frac{\alpha}{\pi} f_{\beta_{c}^{-}}\left(E_{e}, \mu\right)-\frac{\alpha}{\pi} g_{F}\left(E_{e}\right) \frac{E_{e}}{m_{e}}\right)+\frac{1}{M} \frac{1}{1+3 \lambda^{2}}\left[-2 \lambda(\lambda-(\kappa+1)) E_{0}\right.\right. \\
& \left.\left.+\left(4 \lambda^{2}-2(\kappa+1) \lambda-2\right) E_{e}\right]\right\},
\end{aligned}
$$




$$
\begin{aligned}
\tilde{\zeta}\left(E_{e}\right) \tilde{K}\left(E_{e}\right)= & -a_{0}\left(1+\frac{\alpha}{\pi} f_{\beta_{c}^{-}}\left(E_{e}, \mu\right)-\frac{\alpha}{\pi} g_{F}\left(E_{e}\right) \frac{E_{e}}{m_{e}}\right)+\frac{1}{M} \frac{1}{1+3 \lambda^{2}}\left[-2 \lambda(\lambda-(\kappa+1)) E_{0}\right. \\
& \left.+4 \lambda(3 \lambda-(\kappa+1)) E_{e}+\left(8 \lambda^{2}-2(\kappa+1) \lambda+2\right) m_{e}\right] .
\end{aligned}
$$

The use of the Dirac wave function of a free decay electron leads to a vanishing correlation coefficient $\tilde{L}\left(E_{e}\right)=0$. In order to get a non-vanishing correlation coefficient $\tilde{L}\left(E_{e}\right)$ we have to use the Dirac wave function of a decay electron, distorted in the Coulomb field of the decay proton $[28,29,38]$.

\section{CORRELATION COEFFICIENT $L\left(E_{e}\right)$}

For the calculation of the correlation coefficient we use the Dirac wave function of the electron, distorted by the Coulomb proton-electron final state interaction. It is equal to $[28,29,38]$

$$
u_{e}\left(\vec{k}_{e}, \sigma_{e}\right)=\sqrt{\frac{E_{e}+m_{e}(1-\gamma)}{1-\gamma}}\left(\begin{array}{c}
1 \\
\left(1+i \frac{\alpha Z m_{e}}{k_{e}}\right) \frac{\vec{\sigma} \cdot \vec{k}_{e}}{E_{e}+m_{e}(1-\gamma)}
\end{array}\right) \otimes \varphi_{\sigma_{e}},
$$

where $\gamma=1-\sqrt{1-\alpha^{2} Z^{2}}$. The electron wave function Eq. (19) satisfies the Dirac equation [38]

$$
\left(\hat{k}_{e}-m_{e}(1-\gamma)+i \frac{\alpha Z m_{e}}{k_{e}} \gamma^{0} \vec{\gamma} \cdot \vec{k}_{e}\right) u_{e}\left(\vec{k}_{e}, \sigma_{e}\right)=0
$$

We normalize the wave function Eq. (19) in a standard way $\bar{u}_{e}\left(\vec{k}_{e}, \sigma_{e}^{\prime}\right) u_{e}\left(\vec{k}_{e}, \sigma_{e}\right)=2 m_{e} \delta_{\sigma_{e}^{\prime} \sigma_{e}}$. Since $\gamma=O\left(\alpha^{2}\right)$, keeping the contributions of order $O(\alpha)$ we have to set $\gamma=0$. The contribution of the Coulomb distortion to the right-hand side (r.h.s) of Eq. (15), multiplied by the contribution of the phase-volume Eq. (15) is defined by the trace

$$
\begin{aligned}
\Phi_{\beta_{c}^{-}}\left(\vec{k}_{e}, \vec{k}_{\nu}\right) \sum_{\mathrm{pol}} \frac{\left|M\left(n \rightarrow p e^{-} \nu_{e}\right)\right|^{2}}{32 m_{n}^{2} G_{F}^{2}\left|V_{u d}\right|^{2} E_{e} E_{\nu}} \\
=: \frac{1-\lambda^{2}}{1+3 \lambda^{2}} i \frac{\alpha Z m_{e}}{k_{e}} \frac{\operatorname{tr}\left\{\left[\left(\vec{\sigma} \cdot \vec{k}_{e}\right),\left(\vec{\sigma} \cdot \vec{\xi}_{e}\right)\right]\left(\vec{\sigma} \cdot \vec{k}_{\nu}\right)\right\}}{4 E_{e} E_{\nu}} \\
=\frac{1-\lambda^{2}}{1+3 \lambda^{2}} \frac{\alpha Z m_{e}}{k_{e}} \frac{\vec{\xi}_{e} \cdot\left(\vec{k}_{e} \times \vec{k}_{\nu}\right)}{E_{e} E_{\nu}} .
\end{aligned}
$$

We would like to emphasize that the contribution of the Coulomb distortion of the Dirac wave function of a decay electron to the correlation coefficient comes from the traces of $V \times V$ and $A \times A$ products only, i.e., $\operatorname{tr}\{V \times V+A \times A\} \sim$ $\left(1-\lambda^{2}\right)$. Thus, we get

$$
\begin{aligned}
\Phi_{\beta_{c}^{-}}\left(\vec{k}_{e}, \vec{k}_{\nu}\right) \sum_{\mathrm{pol}} \frac{\left|M\left(n \rightarrow p e^{-} \nu_{e}\right)\right|^{2}}{32 m_{n}^{2} G_{F}^{2}\left|V_{u d}\right|^{2} E_{e} E_{\nu}} \\
=\left(1+3 \lambda^{2}\right) \tilde{\zeta}\left(E_{e}\right)\left\{1+\tilde{a}\left(E_{e}\right) \frac{\vec{k}_{e} \cdot \vec{k}_{\nu}}{E_{e} E_{\nu}}+\tilde{G}\left(E_{e}\right) \frac{\vec{\xi}_{e} \cdot \vec{k}_{e}}{E_{e}}+\tilde{H}\left(E_{e}\right) \frac{\vec{\xi}_{e} \cdot \vec{k}_{\nu}}{E_{\nu}}\right. \\
\quad+\tilde{K}_{e}\left(E_{e}\right) \frac{\left(\vec{\xi}_{e} \cdot \vec{k}_{e}\right)\left(\vec{k}_{e} \cdot \vec{k}_{\nu}\right)}{\left(E_{e}+m_{e}\right) E_{e} E_{\nu}}+\tilde{L}\left(E_{e}\right) \frac{\vec{\xi}_{e} \cdot\left(\vec{k}_{e} \times \vec{k}_{\nu}\right)}{E_{e} E_{\nu}}-3 a_{0} \frac{E_{e}}{M}\left(\frac{\left(\vec{k}_{e} \cdot \vec{k}_{\nu}\right)^{2}}{E_{e}^{2} E_{\nu}^{2}}-\frac{1}{3} \frac{k_{e}^{2}}{E_{e}^{2}}\right) \\
\left.\quad+3 a_{0} \frac{m_{e}}{M}\left(\frac{\left(\vec{\xi}_{e} \cdot \vec{k}_{\nu}\right)\left(\vec{k}_{e} \cdot \vec{k}_{\nu}\right)}{E_{e} E_{\nu}^{2}}-\frac{1}{3} \frac{\vec{\xi}_{e} \cdot \vec{k}_{e}}{E_{e}}\right)+3 a_{0} \frac{1}{M}\left(\frac{\left(\vec{\xi}_{e} \cdot \vec{k}_{e}\right)\left(\vec{k}_{e} \cdot \vec{k}_{\nu}\right)^{2}}{\left(E_{e}+m_{e}\right) E_{e} E_{\nu}^{2}}-\frac{1}{3}\left(E_{e}-m_{e}\right) \frac{\vec{\xi}_{e} \cdot \vec{k}_{e}}{E_{e}}\right)\right\} .
\end{aligned}
$$

The correlation coefficient $\tilde{\zeta}\left(E_{e}\right) \tilde{L}\left(E_{e}\right)$ is equal to

$$
\tilde{\zeta}\left(E_{e}\right) \tilde{L}\left(E_{e}\right)=\alpha \frac{m_{e}}{k_{e}} a_{0},
$$


where we have set $Z=1$. Thus, the electron-energy and electron-antineutrino angular distribution of the neutron $\beta^{-}$-decay with polarized electron and unpolarized neutron and proton is

$$
\begin{aligned}
& \frac{d^{5} \lambda_{\beta_{c}^{-}}\left(E_{e}, \vec{k}_{e}, \vec{\xi}_{e}, \vec{k}_{\nu}\right)}{d E_{e} d \Omega_{e} d \Omega_{\nu}} \\
& =\left(1+3 \lambda^{2}\right) \frac{G_{F}^{2}\left|V_{u d}\right|^{2}}{32 \pi^{5}}\left(E_{0}-E_{e}\right)^{2} \sqrt{E_{e}^{2}-m_{e}^{2}} E_{e} F\left(E_{e}, Z=1\right) \tilde{\zeta}\left(E_{e}\right)\left\{1+\tilde{a}\left(E_{e}\right) \frac{\vec{k}_{e} \cdot \vec{k}_{\nu}}{E_{e} E_{\nu}}\right. \\
& \quad+\tilde{G}\left(E_{e}\right) \frac{\vec{\xi}_{e} \cdot \vec{k}_{e}}{E_{e}}+\tilde{H}\left(E_{e}\right) \frac{\vec{\xi}_{e} \cdot \vec{k}_{\nu}}{E_{\nu}}+\tilde{K}_{e}\left(E_{e}\right) \frac{\left(\vec{\xi}_{e} \cdot \vec{k}_{e}\right)\left(\vec{k}_{e} \cdot \vec{k}_{\nu}\right)}{\left(E_{e}+m_{e}\right) E_{e} E_{\nu}}+\tilde{L}\left(E_{e}\right) \frac{\vec{\xi}_{e} \cdot\left(\vec{k}_{e} \times \vec{k}_{\nu}\right)}{E_{e} E_{\nu}}-3 a_{0} \frac{E_{e}}{M}\left(\frac{\left(\vec{k}_{e} \cdot \vec{k}_{\nu}\right)^{2}}{E_{e}^{2} E_{\nu}^{2}}-\frac{1}{3} \frac{k_{e}^{2}}{E_{e}^{2}}\right) \\
& \left.\quad+3 a_{0} \frac{m_{e}}{M}\left(\frac{\left(\vec{\xi}_{e} \cdot \vec{k}_{\nu}\right)\left(\vec{k}_{e} \cdot \vec{k}_{\nu}\right)}{E_{e} E_{\nu}^{2}}-\frac{1}{3} \frac{\vec{\xi}_{e} \cdot \vec{k}_{e}}{E_{e}}\right)+3 a_{0} \frac{1}{M}\left(\frac{\left(\vec{\xi}_{e} \cdot \vec{k}_{e}\right)\left(\vec{k}_{e} \cdot \vec{k}_{\nu}\right)^{2}}{\left(E_{e}+m_{e}\right) E_{e} E_{\nu}^{2}}-\frac{1}{3}\left(E_{e}-m_{e}\right) \frac{\vec{\xi}_{e} \cdot \vec{k}_{e}}{E_{e}}\right)\right\} .
\end{aligned}
$$

The radiative corrections to the correlation coefficients, defined by the function $f_{\beta_{c}^{-}}\left(E_{e}, \mu\right)$, depend on the infrared cutoff $\mu$. In order to remove such a dependence we have to add the contribution of the neutron radiative $\beta^{-}$-decay [5] (see also [1,2]).

\section{ELECTRON-ENERGY AND ELECTRON-ANTINEUTRINO ANGULAR DISTRIBUTION OF NEUTRON $\beta^{-}$-DECAY WITH POLARIZED ELECTRON AND UNPOLARIZED NEUTRON AND PROTON TO ORDER $10^{-3}$}

Summing the electron-energy and electron-antineutrino angular distributions Eq. (24) and Eq. (A5) in the Appendix we obtain the electron-energy and electron-antineutrino angular distribution of $\lambda_{n}=\lambda_{\beta_{c}^{-}}+\lambda_{\beta^{-} \gamma}$ equal to

$$
\begin{aligned}
& \frac{d^{5} \lambda_{n}\left(E_{e}, \vec{k}_{e}, \vec{\xi}_{e}, \vec{k}_{\nu}\right)}{d E_{e} d \Omega_{e} d \Omega_{\nu}} \\
& =\left(1+3 \lambda^{2}\right) \frac{G_{F}^{2}\left|V_{u d}\right|^{2}}{32 \pi^{5}}\left(E_{0}-E_{e}\right)^{2} \sqrt{E_{e}^{2}-m_{e}^{2}} E_{e} F\left(E_{e}, Z=1\right) \zeta\left(E_{e}\right)\left\{1+a\left(E_{e}\right) \frac{\vec{k}_{e} \cdot \vec{k}_{\nu}}{E_{e} E_{\nu}}\right. \\
& \quad+G\left(E_{e}\right) \frac{\vec{\xi}_{e} \cdot \vec{k}_{e}}{E_{e}}+H\left(E_{e}\right) \frac{\vec{\xi}_{e} \cdot \vec{k}_{\nu}}{E_{\nu}}+K_{e}\left(E_{e}\right) \frac{\left(\vec{\xi}_{e} \cdot \vec{k}_{e}\right)\left(\vec{k}_{e} \cdot \vec{k}_{\nu}\right)}{\left(E_{e}+m_{e}\right) E_{e} E_{\nu}}+L\left(E_{e}\right) \frac{\vec{\xi}_{e} \cdot\left(\vec{k}_{e} \times \vec{k}_{\nu}\right)}{E_{e} E_{\nu}}-3 a_{0} \frac{E_{e}}{M}\left(\frac{\left(\vec{k}_{e} \cdot \vec{k}_{\nu}\right)^{2}}{E_{e}^{2} E_{\nu}^{2}}-\frac{1}{3} \frac{k_{e}^{2}}{E_{e}^{2}}\right) \\
& \left.\quad+3 a_{0} \frac{m_{e}}{M}\left(\frac{\left(\vec{\xi}_{e} \cdot \vec{k}_{\nu}\right)\left(\vec{k}_{e} \cdot \vec{k}_{\nu}\right)}{E_{e} E_{\nu}^{2}}-\frac{1}{3} \frac{\vec{\xi}_{e} \cdot \vec{k}_{e}}{E_{e}}\right)+3 a_{0} \frac{1}{M}\left(\frac{\left(\vec{\xi}_{e} \cdot \vec{k}_{e}\right)\left(\vec{k}_{e} \cdot \vec{k}_{\nu}\right)^{2}}{\left(E_{e}+m_{e}\right) E_{e} E_{\nu}^{2}}-\frac{1}{3}\left(E_{e}-m_{e}\right) \frac{\vec{\xi}_{e} \cdot \vec{k}_{e}}{E_{e}}\right)\right\} .
\end{aligned}
$$

The correlation coefficients are equal to

$$
\begin{aligned}
\zeta\left(E_{e}\right)= & \left(1+\frac{\alpha}{\pi} g_{n}\left(E_{e}\right)\right)+\frac{1}{M} \frac{1}{1+3 \lambda^{2}}\left[-2 \lambda(\lambda-(\kappa+1)) E_{0}+\left(10 \lambda^{2}-4(\kappa+1) \lambda+2\right) E_{e}-2 \lambda(\lambda-(\kappa+1)) \frac{m_{e}^{2}}{E_{e}}\right], \\
\zeta\left(E_{e}\right) a\left(E_{e}\right)= & a_{0}\left(1+\frac{\alpha}{\pi} g_{n}\left(E_{e}\right)+\frac{\alpha}{\pi} f_{n}\left(E_{e}\right)\right)+\frac{1}{M} \frac{1}{1+3 \lambda^{2}}\left[2 \lambda(\lambda-(\kappa+1)) E_{0}-4 \lambda(3 \lambda-(\kappa+1)) E_{e}\right], \\
\zeta\left(E_{e}\right) G\left(E_{e}\right)= & -\left(1+\frac{\alpha}{\pi} g_{n}\left(E_{e}\right)+\frac{\alpha}{\pi} f_{n}\left(E_{e}\right)\right)+\frac{1}{M} \frac{1}{1+3 \lambda^{2}}\left[2 \lambda(\lambda-(\kappa+1)) E_{0}-\left(10 \lambda^{2}-4(\kappa+1) \lambda+2\right) E_{e}\right] \\
\zeta\left(E_{e}\right) H\left(E_{e}\right)= & \frac{m_{e}}{E_{e}}\left\{-a_{0}\left(1+\frac{\alpha}{\pi} g_{n}\left(E_{e}\right)+\frac{\alpha}{\pi} h_{n}^{(3)}\left(E_{e}\right)\right)+\frac{1}{M} \frac{1}{1+3 \lambda^{2}}\left[-2 \lambda(\lambda-(\kappa+1)) E_{0}+\left(4 \lambda^{2}-2(\kappa+1) \lambda-2\right) E_{e}\right]\right\}, \\
\zeta\left(E_{e}\right) K_{e}\left(E_{e}\right)= & -a_{0}\left(1+\frac{\alpha}{\pi} g_{n}\left(E_{e}\right)+\frac{\alpha}{\pi} h_{n}^{(4)}\left(E_{e}\right)\right)+\frac{1}{M} \frac{1}{1+3 \lambda^{2}}\left[-2 \lambda(\lambda-(\kappa+1)) E_{0}+4 \lambda(3 \lambda-(\kappa+1)) E_{e}\right. \\
& \left.+\left(8 \lambda^{2}-2(\kappa+1) \lambda+2\right) m_{e}\right], \\
\zeta\left(E_{e}\right) L\left(E_{e}\right)= & \alpha \frac{m_{e}}{k_{e}} a_{0}
\end{aligned}
$$

The radiative corrections of order $O(\alpha / \pi)$ to the correlation coefficients are defined by the functions $g_{n}\left(E_{e}\right), f_{n}\left(E_{e}\right)$ and the functions $h_{n}^{(3)}\left(E_{e}\right)$ and $h_{n}^{(4)}\left(E_{e}\right)$. The functions $g_{n}\left(E_{e}\right)$ and $f_{n}\left(E_{e}\right)$ have been calculated by Sirlin [5] and Shann [6] 


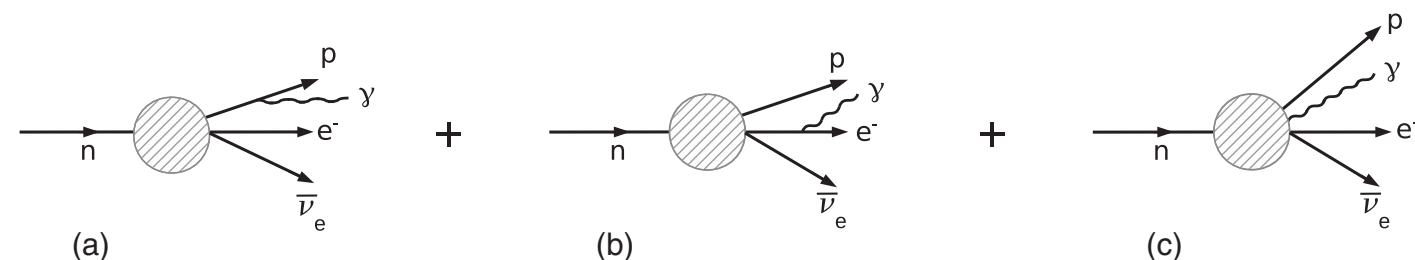

FIG. 2. The Feynman diagrams, defining the contribution to the amplitude of the neutron radiative $\beta^{-}$-decay in the tree-approximation to order $e$.
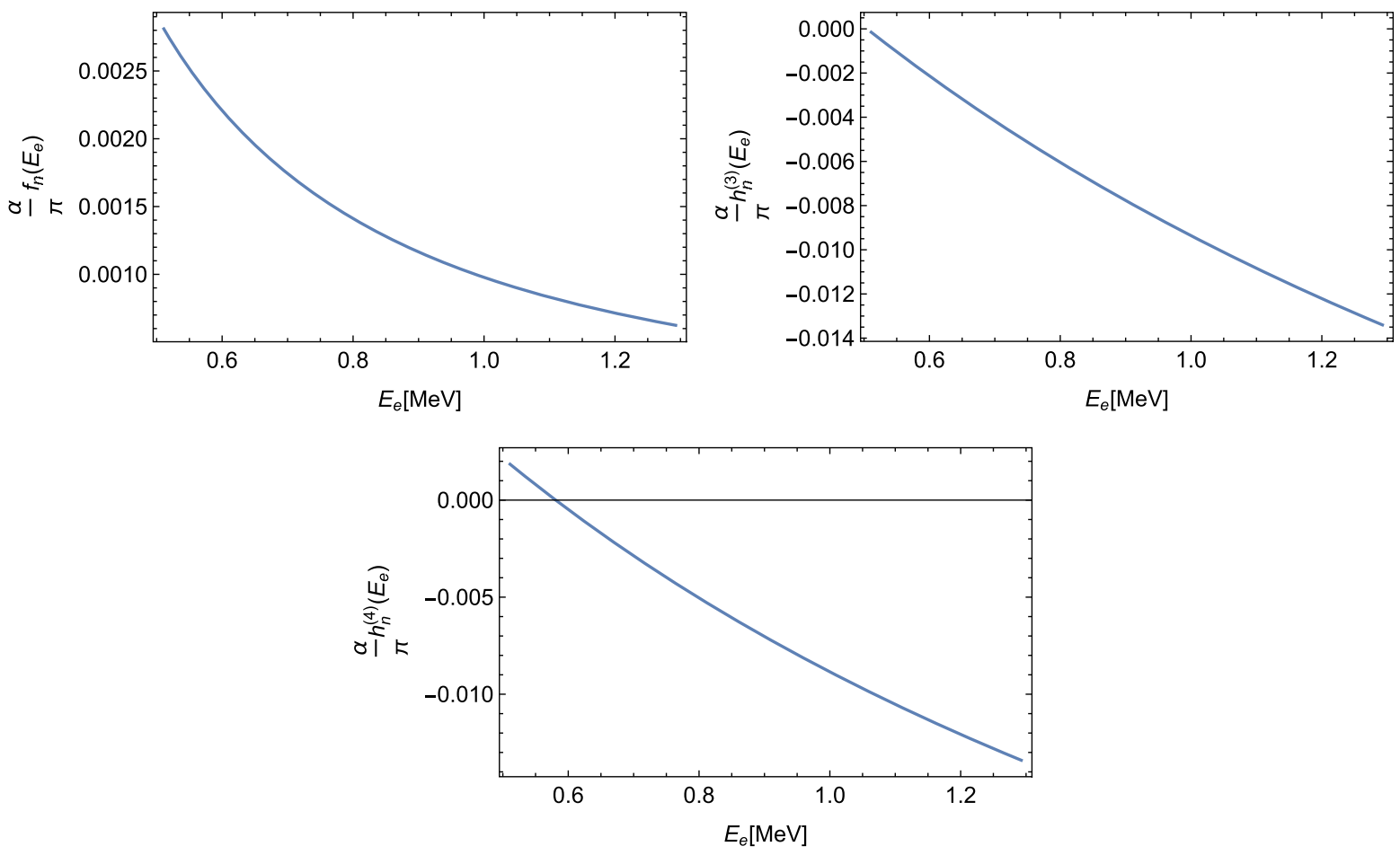

FIG. 3. Radiative corrections $(\alpha / \pi) f_{n}\left(E_{e}\right),(\alpha / \pi) h_{n}^{(3)}\left(E_{e}\right)$, and $(\alpha / \pi) h_{n}^{(4)}\left(E_{e}\right)$ to the correlation coefficients $G\left(E_{e}\right), H\left(E_{e}\right)$ and $K_{e}\left(E_{e}\right)$ of the electron-antineutrino energy and angular distribution Eq. (25).

(see also [7] and Appendices B, C, D, E and F in Ref. [1]), respectively. The contributions of the electroweak-boson exchanges and QCD corrections to the function $g_{n}\left(E_{e}\right)$ have been calculated in [35-37]. In turn, the radiative corrections $(\alpha / \pi) h_{n}^{(3)}\left(E_{e}\right)$ and $(\alpha / \pi) h_{n}^{(4)}\left(E_{e}\right)$ are calculated in Appendix A. The functions $(\alpha / \pi) h_{n}^{(3)}\left(E_{e}\right)$ and $(\alpha / \pi) h_{n}^{(4)}\left(E_{e}\right)$, together with the function $(\alpha / \pi) f_{n}\left(E_{e}\right)$, are plotted in Fig. 3 in the electron-energy region $m_{e} \leq E_{e} \leq E_{0}$.

\section{CORRELATION COEFFICIENTS $a\left(E_{e}\right), G\left(E_{e}\right), H\left(E_{e}\right)$, AND $K_{e}\left(E_{e}\right)$ TO ORDER 10-3}

The correlation coefficients $a\left(E_{e}\right)$ and $G\left(E_{e}\right)$ have been calculated in [1,2], respectively. They are equal to

$$
\begin{aligned}
a\left(E_{e}\right)= & \left(1+\frac{\alpha}{\pi} f_{n}\left(E_{e}\right)\right)\left\{a_{0}+\frac{1}{M} \frac{1}{1+3 \lambda^{2}}\left[2 \lambda(\lambda-(\kappa+1)) E_{0}-4 \lambda(3 \lambda-(\kappa+1)) E_{e}\right]\right. \\
& \left.+\frac{1}{M} \frac{a_{0}}{1+3 \lambda^{2}}\left[-\left(10 \lambda^{2}-4(\kappa+1) \lambda+2\right) E_{e}+\left(2 \lambda^{2}-2(\kappa+1) \lambda\right)\left(E_{0}+\frac{m_{e}^{2}}{E_{e}}\right)\right]\right\}, \\
G\left(E_{e}\right)= & -\left(1+\frac{\alpha}{\pi} f_{n}\left(E_{e}\right)\right)\left(1+\frac{1}{M} \frac{1}{1+3 \lambda^{2}}\left(2 \lambda^{2}-2(\kappa+1) \lambda\right) \frac{m_{e}^{2}}{E_{e}}\right) .
\end{aligned}
$$

For the correlation coefficients $H\left(E_{e}\right)$ and $K_{e}\left(E_{e}\right)$ we obtain the following expressions 


$$
\begin{aligned}
H\left(E_{e}\right)= & \left(1+\frac{\alpha}{\pi} h_{n}^{(3)}\left(E_{e}\right)\right) \frac{m_{e}}{E_{e}}\left\{-a_{0}+\frac{1}{M} \frac{1}{1+3 \lambda^{2}}\left[-2 \lambda(\lambda-(\kappa+1)) E_{0}+\left(4 \lambda^{2}-2(\kappa+1) \lambda-2\right) E_{e}\right]\right. \\
& \left.-\frac{1}{M} \frac{a_{0}}{1+3 \lambda^{2}}\left[-\left(10 \lambda^{2}-4(\kappa+1) \lambda+2\right) E_{e}+\left(2 \lambda^{2}-2(\kappa+1) \lambda\right)\left(E_{0}+\frac{m_{e}^{2}}{E_{e}}\right)\right]\right\}
\end{aligned}
$$

and

$$
\begin{aligned}
K_{e}\left(E_{e}\right)= & \left(1+\frac{\alpha}{\pi} h_{n}^{(4)}\left(E_{e}\right)\right)\left\{-a_{0}+\frac{1}{M} \frac{1}{1+3 \lambda^{2}}\left[-2 \lambda(\lambda-(\kappa+1)) E_{0}+4 \lambda(3 \lambda-(\kappa+1)) E_{e}+\left(8 \lambda^{2}-2(\kappa+1) \lambda+2\right) m_{e}\right]\right. \\
& \left.-\frac{1}{M} \frac{a_{0}}{1+3 \lambda^{2}}\left[-\left(10 \lambda^{2}-4(\kappa+1) \lambda+2\right) E_{e}+\left(2 \lambda^{2}-2(\kappa+1) \lambda\right)\left(E_{0}+\frac{m_{e}^{2}}{E_{e}}\right)\right]\right\}
\end{aligned}
$$

The obtained correlation coefficients are calculated to order $10^{-3}$, taking into account the complete set of corrections of order $O\left(E_{e} / M\right)$ and $O(\alpha / \pi)$, caused by the weak magnetism, proton recoil, and one-photon exchanges, respectively.
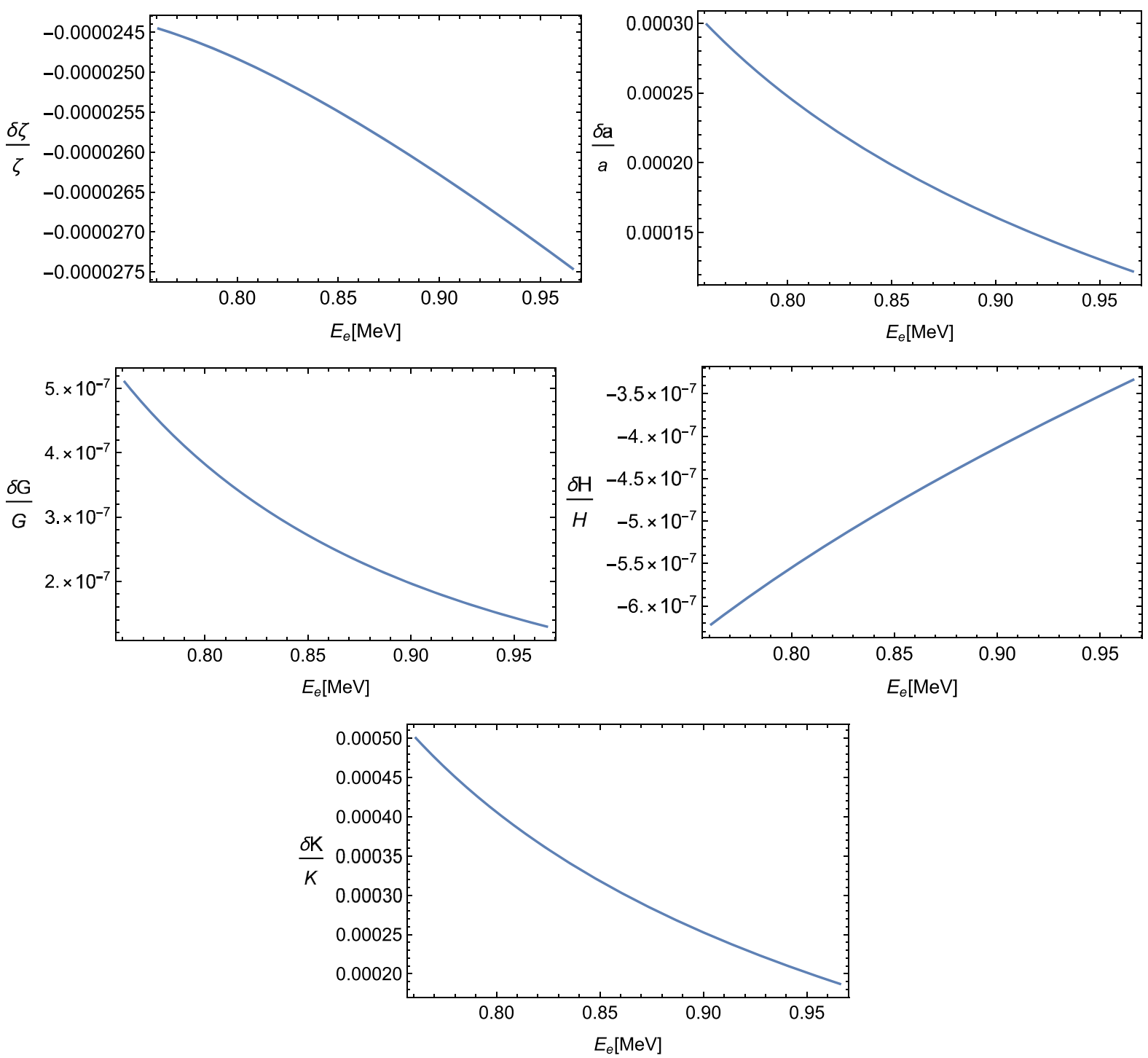

FIG. 4. Relative corrections to the correlation coefficients $\zeta\left(E_{e}\right), a\left(E_{e}\right), G\left(E_{e}\right), H\left(E_{e}\right)$, and $K_{e}\left(E_{e}\right)$ induced by the proton recoil to the Fermi function, caused by the Coulomb electron-proton final-state interaction and calculated for the experimentally observable electron energy region $0.761 \mathrm{MeV} \leq E_{e} \leq 0.966 \mathrm{MeV}[1]$. 


\section{WILKINSON'S CORRECTIONS}

According to Wilkinson [8], the higher order corrections with respect to those calculated in Sec. VII should be caused by (i) the proton recoil in the Coulomb electronproton final-state interaction, (ii) the finite proton radius, (iii) the proton-lepton convolution, and (iv) the higherorder outer radiative corrections.

The relative corrections to the correlation coefficients $\zeta\left(E_{e}\right), a\left(E_{e}\right), G\left(E_{e}\right), H\left(E_{e}\right)$, and $K_{e}\left(E_{e}\right)$, caused by the proton recoil in the final state electron-proton Coulomb interactions, are equal to

$$
\begin{aligned}
\frac{\delta \zeta\left(E_{e}\right)}{\zeta\left(E_{e}\right)}= & -\frac{\pi \alpha}{\beta} \frac{E_{e}}{M}-\frac{1}{3} \frac{1-\lambda^{2}}{1+3 \lambda^{2}} \frac{\pi \alpha}{\beta} \frac{E_{0}-E_{e}}{M}, \\
\frac{\delta a\left(E_{e}\right)}{a\left(E_{e}\right)}= & \frac{1}{3} \frac{1-\lambda^{2}}{1+3 \lambda^{2}} \frac{\pi \alpha}{\beta} \frac{E_{0}-E_{e}}{M}-\frac{1+3 \lambda^{2}}{1-\lambda^{2}} \frac{\pi \alpha}{\beta^{3}} \frac{E_{0}-E_{e}}{M}, \\
\frac{\delta G\left(E_{e}\right)}{G\left(E_{e}\right)}= & -\frac{1}{3} \frac{1-\lambda^{2}}{1+3 \lambda^{2}}\left(1-\beta^{2}\right) \frac{\pi \alpha}{\beta^{3}} \frac{E_{0}-E_{e}}{M}, \\
\frac{\delta H\left(E_{e}\right)}{H\left(E_{e}\right)}= & \frac{1}{3} \frac{1-\lambda^{2}}{1+3 \lambda^{2}} \frac{\pi \alpha}{\beta} \frac{E_{0}-E_{e}}{M}, \\
\frac{\delta K_{e}\left(E_{e}\right)}{K_{e}\left(E_{e}\right)}= & \frac{1}{3} \frac{1-\lambda^{2}}{1+3 \lambda^{2}} \frac{\pi \alpha}{\beta} \frac{E_{0}-E_{e}}{M} \\
& -\frac{1+3 \lambda^{2}}{1-\lambda^{2}} \frac{\pi \alpha}{\beta^{3}} \frac{E_{0}-E_{e}}{M}\left(1+\sqrt{1-\beta^{2}}\right) .
\end{aligned}
$$

In the experimental electron energy region $0.761 \mathrm{MeV} \leq$ $E_{e} \leq 0.966 \mathrm{MeV}$ the corrections Eq. (30) are plotted in Fig. 4 and take the values adduced in Table I. The proton recoil corrections to the correlation coefficient $a\left(E_{e}\right)$, caused by the electron-proton final-state Coulomb interactions, are
TABLE I. Wilkinson's corrections, induced by the change of the Fermi function caused by the electron-proton final-state Coulomb interaction, in the energy region $0.761 \mathrm{MeV} \leq E_{e} \leq$ $0.966 \mathrm{MeV}$.

\begin{tabular}{lcc}
\hline \hline$E_{e}=0.761 \mathrm{MeV}$ & $\delta X\left(E_{e}\right) / X\left(E_{e}\right)$ & $E_{e}=0.966 \mathrm{MeV}$ \\
\hline$-2.5 \times 10^{-5}$ & $\geq \delta \zeta\left(E_{e}\right) / \zeta\left(E_{e}\right) \geq$ & $-2.8 \times 10^{-5}$ \\
$+3.0 \times 10^{-4}$ & $\geq \delta a\left(E_{e}\right) / a\left(E_{e}\right) \geq$ & $+1.1 \times 10^{-4}$ \\
$+5.1 \times 10^{-7}$ & $\geq \delta G\left(E_{e}\right) / G\left(E_{e}\right) \geq$ & $+1.3 \times 10^{-7}$ \\
$-6.2 \times 10^{-7}$ & $\leq \delta H\left(E_{e}\right) / H\left(E_{e}\right) \leq$ & $-3.3 \times 10^{-7}$ \\
$+5.0 \times 10^{-4}$ & $\geq \delta K_{e}\left(E_{e}\right) / K_{e}\left(E_{e}\right) \geq$ & $+1.9 \times 10^{-4}$ \\
\hline \hline
\end{tabular}

of order $10^{-4}$ and should be taken into account for the analysis of the experimental data on searches of contributions of interactions beyond the SM at the level of $10^{-4}$ [21].

In turn, Wilkinson's corrections, caused by (ii) the finite proton radius, (iii) the proton-lepton convolution, and (iv) the higher-order outer radiative corrections, retain their expression for calculated in [2] and the order $\left|\delta \zeta\left(E_{e}\right) / \zeta\left(E_{e}\right)\right| \sim$ $10^{-5}, \quad\left|\delta a\left(E_{e}\right) / a\left(E_{e}\right)\right| \sim\left|\delta K_{e}\left(E_{e}\right) / K_{e}\left(E_{e}\right)\right| \sim 10^{-4}$, and $\left|\delta G\left(E_{e}\right) / G\left(E_{e}\right)\right| \sim\left|\delta H\left(E_{e}\right) / H\left(E_{e}\right)\right| \sim 10^{-7}$, respectively.

\section{ELECTRON-ENERGY AND ELECTRON- ANTINEUTRINO ANGULAR DISTRIBUTION BEYOND THE SM}

For the calculation of contributions of interactions beyond the SM we use the effective low-energy Hamiltonian of weak nucleon-lepton four-fermion local interactions, taking into account all phenomenological couplings beyond the SM [9-20] in the notations of [1,3]:

$$
\begin{aligned}
\mathcal{H}_{W}(x)= & \frac{G_{F}}{\sqrt{2}} V_{u d}\left\{\left[\bar{\psi}_{p}(x) \gamma_{\mu} \psi_{n}(x)\right]\left[\bar{\psi}_{e}(x) \gamma^{\mu}\left(C_{V}+\bar{C}_{V} \gamma^{5}\right) \psi_{\nu_{e}}(x)\right]+\left[\bar{\psi}_{p}(x) \gamma_{\mu} \gamma^{5} \psi_{n}(x)\right]\left[\bar{\psi}_{e}(x) \gamma^{\mu}\left(\bar{C}_{A}+C_{A} \gamma^{5}\right) \psi_{\nu_{e}}(x)\right]\right. \\
& +\left[\bar{\psi}_{p}(x) \psi_{n}(x)\right]\left[\bar{\psi}_{e}(x)\left(C_{S}+\bar{C}_{S} \gamma^{5}\right) \psi_{\nu_{e}}(x)\right]+\left[\bar{\psi}_{p}(x) \gamma^{5} \psi_{n}(x)\right]\left[\bar{\psi}_{e}(x)\left(C_{P}+\bar{C}_{P} \gamma^{5}\right) \psi_{\nu_{e}}(x)\right] \\
& +\frac{1}{2}\left[\bar{\psi}_{p}(x) \sigma^{\mu \nu} \psi_{n}(x)\right]\left[\bar{\psi}_{e}(x) \sigma_{\mu \nu}\left(C_{T}+\bar{C}_{T} \gamma^{5}\right) \psi_{\nu_{e}}(x)\right\}
\end{aligned}
$$

This is the most general form of the effective low-energy weak interactions, where the phenomenological coupling constants $C_{i}$ and $\bar{C}_{i}$ for $i=V, A, S, P$, and $T$ can be induced by the left-handed and right-handed hadronic and leptonic currents [9-14]. They are related to the phenomenological coupling constants, analogous to those which were introduced by Herczeg [13], as follows

$$
\begin{array}{ll}
C_{V}=1+a_{L L}^{h}+a_{L R}^{h}+a_{R R}^{h}+a_{R L}^{h}, & \bar{C}_{V}=-1-a_{L L}^{h}-a_{L R}^{h}+a_{R R}^{h}+a_{R L}^{h}, \quad C_{A}=-\lambda+a_{L L}^{h}-a_{L R}^{h}+a_{R R}^{h}-a_{R L}^{h}, \\
\bar{C}_{A}=\lambda-a_{L L}^{h}+a_{L R}^{h}+a_{R R}^{h}-a_{R L}^{h}, & C_{S}=A_{L L}^{h}+A_{L R}^{h}+A_{R R}^{h}+A_{R L}^{h}, \quad \bar{C}_{S}=-A_{L L}^{h}-A_{L R}^{h}+A_{R R}^{h}+A_{R L}^{h}, \\
C_{P}=-A_{L L}^{h}+A_{L R}^{h}+A_{R R}^{h}-A_{R L}^{h}, & \bar{C}_{P}=A_{L L}^{h}-A_{L R}^{h}+A_{R R}^{h}-A_{R L}^{h}, \quad C_{T}=2\left(\alpha_{L L}^{h}+\alpha_{R R}^{h}\right), \quad \bar{C}_{T}=2\left(-\alpha_{L L}^{h}+\alpha_{R R}^{h}\right),
\end{array}
$$

where the index $h$ means that the phenomenological coupling constants are introduced at the hadronic level but not at the quark level as it has been done by Herczeg [13]. In the SM the phenomenological coupling constants $C_{i}$ and $\bar{C}_{i}$ for $i=V, A$, $S, P$ and $T$ are equal to $C_{S}=\bar{C}_{S}=C_{P}=\bar{C}_{P}=C_{T}=\bar{C}_{T}=0, C_{V}=-\bar{C}_{V}=1$ and $C_{A}=-\bar{C}_{A}=-\lambda$ [1]. The phenomenological coupling constants $a_{i j}^{h}, A_{i j}^{h}$ and $\alpha_{j j}^{h}$ for $i(j)=L$ or $R$ are induced by interactions beyond the SM.

The contribution of interactions beyond the SM, given by the Hamiltonian of weak interactions Eq. (6), to the amplitude of the neutron $\beta^{-}$-decay, calculated to leading order in the large nucleon mass expansion, takes the form 


$$
\begin{aligned}
M\left(n \rightarrow p e^{-} \bar{\nu}_{e}\right)= & -2 m_{n} \frac{G_{F}}{\sqrt{2}} V_{u d}\left\{\left[\varphi_{p}^{\dagger} \varphi_{n}\right]\left[\bar{u}_{e} \gamma^{0}\left(C_{V}+\bar{C}_{V} \gamma^{5}\right) v_{\bar{\nu}}\right]-\left[\varphi_{p}^{\dagger} \vec{\sigma} \varphi_{n}\right] \cdot\left[\bar{u}_{e} \vec{\gamma}\left(\bar{C}_{A}+C_{A} \gamma^{5}\right) v_{\bar{\nu}}\right]\right. \\
& \left.+\left[\varphi_{p}^{\dagger} \varphi_{n}\right]\left[\bar{u}_{e}\left(C_{S}+\bar{C}_{S} \gamma^{5}\right) v_{\bar{\nu}}\right]+\left[\varphi_{p}^{\dagger} \vec{\sigma} \varphi_{n}\right] \cdot\left[\bar{u}_{e} \gamma^{0} \vec{\gamma}\left(\bar{C}_{T}+C_{T} \gamma^{5}\right) v_{\bar{\nu}}\right]\right\} .
\end{aligned}
$$

The Hermitian conjugate amplitude is

$$
\begin{aligned}
M^{\dagger}\left(n \rightarrow p e^{-} \bar{\nu}_{e}\right)= & -2 m_{n} \frac{G_{F}}{\sqrt{2}} V_{u d}^{*}\left\{\left[\varphi_{n}^{\dagger} \varphi_{p}\right]\left[\bar{v}_{\bar{\nu}} \gamma^{0}\left(C_{V}^{*}+\bar{C}_{V}^{*} \gamma^{5}\right) u_{e}\right]-\left[\varphi_{n}^{\dagger} \vec{\sigma} \varphi_{p}\right] \cdot\left[\bar{v}_{\bar{\nu}} \vec{\gamma}\left(\bar{C}_{A}^{*}+C_{A}^{*} \gamma^{5}\right) u_{e}\right]\right. \\
& \left.+\left[\varphi_{n}^{\dagger} \varphi_{p}\right]\left[\bar{v}_{\bar{\nu}}\left(C_{S}^{*}-\bar{C}_{S}^{*} \gamma^{5}\right) u_{e}\right]-\left[\varphi_{n}^{\dagger} \vec{\sigma} \varphi_{p}\right] \cdot\left[\bar{v}_{\bar{\nu}} \gamma^{0} \vec{\gamma}\left(\bar{C}_{T}^{*}-C_{T}^{*} \gamma^{5}\right) u_{e}\right]\right\} .
\end{aligned}
$$

The contributions of interactions with the strength, defined by the phenomenological coupling constants $C_{P}$ and $\bar{C}_{P}$, may appear only of order $O\left(C_{P} E_{e} / M\right)$ and $O\left(\bar{C}_{P} E_{e} / M\right)$ and can be neglected to leading order in the large nucleon mass expansion. We have also neglected the contributions of the neutron-proton mass difference. The squared absolute value of the amplitude Eq. (8), summed over polarizations of massive fermions, is equal to

$$
\begin{aligned}
\sum_{\text {pol. }} & \frac{\left|M\left(n \rightarrow p e^{-} \bar{\nu}_{e}\right)\right|^{2}}{8 m_{n}^{2} G_{F}^{2}\left|V_{u d}\right|^{2} E_{\nu} E_{e}} \\
= & \left\{\frac{1}{2}\left(\left|C_{V}\right|^{2}+\left|\bar{C}_{V}\right|^{2}+3\left|C_{A}\right|^{2}+3\left|\bar{C}_{A}\right|^{2}+\left|C_{S}\right|^{2}+\left|\bar{C}_{S}\right|^{2}+3\left|C_{T}\right|^{2}+3\left|\bar{C}_{T}\right|^{2}\right)+\frac{m_{e}}{E_{e}} \operatorname{Re}\left(C_{V} C_{S}^{*}+\bar{C}_{V} \bar{C}_{S}^{*}-3 C_{A} C_{T}^{*}-3 \bar{C}_{A} \bar{C}_{T}^{*}\right)\right. \\
& +\frac{\vec{k}_{e} \cdot \vec{k}_{\nu}}{E_{e} E_{\nu}} \frac{1}{2}\left(\left|C_{V}\right|^{2}+\left|\bar{C}_{V}\right|^{2}-\left|C_{A}\right|^{2}-\left|\bar{C}_{A}\right|^{2}-\left|C_{S}\right|^{2}-\left|\bar{C}_{S}\right|^{2}+\left|C_{T}\right|^{2}+\left|\bar{C}_{T}\right|^{2}\right)+\frac{\vec{\xi}_{e} \cdot \vec{k}_{e}}{E_{e}} \operatorname{Re}\left(C_{V} \bar{C}_{V}^{*}+3 C_{A} \bar{C}_{A}^{*}-C_{S} \bar{C}_{S}^{*}\right. \\
& \left.-3 C_{T} \bar{C}_{T}^{*}\right)+\frac{\vec{\xi}_{e} \cdot \vec{k}_{\nu}}{E_{\nu}} \operatorname{Re}\left(C_{V} \bar{C}_{S}^{*}+\bar{C}_{V} C_{S}^{*}+C_{A} \bar{C}_{T}^{*}+\bar{C}_{A} C_{T}^{*}+\frac{m_{e}}{E_{e}}\left(C_{V} \bar{C}_{V}^{*}-C_{A} \bar{C}_{A}^{*}+C_{S} \bar{C}_{S}^{*}-C_{T} \bar{C}_{T}^{*}\right)\right) \\
& +\frac{\left(\vec{\xi}_{e} \cdot \vec{k}_{e}\right)\left(\vec{k}_{e} \cdot \vec{k}_{\nu}\right)}{\left(E_{e}+m_{e}\right) E_{e} E_{\nu}} \operatorname{Re}\left(C_{V} \bar{C}_{V}^{*}-C_{A} \bar{C}_{A}^{*}+C_{S} \bar{C}_{S}^{*}-C_{T} \bar{C}_{T}^{*}-C_{V} \bar{C}_{S}^{*}-\bar{C}_{V} C_{S}^{*}-C_{A} \bar{C}_{T}^{*}-\bar{C}_{A} C_{T}^{*}\right) \\
& \left.+\frac{\vec{\xi}_{e} \cdot\left(\vec{k}_{e} \times \vec{k}_{\nu}\right)}{E_{e} E_{\nu}} \operatorname{Im}\left(C_{S} C_{V}^{*}+\bar{C}_{S} \bar{C}_{V}^{*}+C_{T} C_{A}^{*}+\bar{C}_{T} \bar{C}_{A}^{*}\right)\right\} .
\end{aligned}
$$

The structure of the correlation coefficients in Eq. (35) agrees well with the structure of the corresponding expressions obtained in [11]. In the linear approximation for coupling constants of vector and axial-vector interactions beyond the SM [1] we get

$$
\begin{aligned}
\sum_{\text {pol. }} & \frac{\left|M\left(n \rightarrow p e^{-} \bar{\nu}_{e}\right)\right|^{2}}{8 m_{n}^{2} G_{F}^{2}\left|V_{u d}\right|^{2} E_{\nu} E_{e}\left(1+3 \lambda^{2}\right)} \\
= & \left\{\left[1+\frac{1}{2} \frac{1}{1+3 \lambda^{2}}\left(\left|C_{S}\right|^{2}+\left|\bar{C}_{S}\right|^{2}+3\left|C_{T}\right|^{2}+3\left|\bar{C}_{T}\right|^{2}\right)\right]+\frac{m_{e}}{E_{e}} \frac{1}{1+3 \lambda^{2}} \operatorname{Re}\left(\left(C_{S}-\bar{C}_{S}\right)+3 \lambda\left(C_{T}-\bar{C}_{T}\right)\right)\right. \\
& +\frac{\vec{k}_{e} \cdot \vec{k}_{\nu}}{E_{e} E_{\nu}}\left[a_{0}-\frac{1}{2} \frac{1}{1+3 \lambda^{2}}\left(\left|C_{S}\right|^{2}+\left|\bar{C}_{S}\right|^{2}-\left|C_{T}\right|^{2}-\left|\bar{C}_{T}\right|^{2}\right)\right]+\frac{\vec{k}_{e} \cdot \vec{\xi}_{e}}{E_{e}}\left[-1-\frac{1}{1+3 \lambda^{2}} \operatorname{Re}\left(C_{S} \bar{C}_{S}^{*}+3 C_{T} \bar{C}_{T}^{*}\right)\right] \\
& +\frac{\vec{\xi}_{e} \cdot \vec{k}_{\nu}}{E_{\nu}}\left[-\frac{m_{e}}{E_{e}} a_{0}-\frac{1}{1+3 \lambda^{2}} \operatorname{Re}\left(\left(C_{S}-\bar{C}_{S}\right)-\lambda\left(C_{T}-\bar{C}_{T}\right)\right)+\frac{m_{e}}{E_{e}} \frac{1}{1+3 \lambda^{2}} \operatorname{Re}\left(C_{S} \bar{C}_{S}^{*}-C_{T} \bar{C}_{T}^{*}\right)\right] \\
& +\frac{\left(\vec{\xi}_{e} \cdot \vec{k}_{e}\right)\left(\vec{k}_{e} \cdot \vec{k}_{\nu}\right)}{\left(E_{e}+m_{e}\right) E_{e} E_{\nu}}\left[-a_{0}+\frac{1}{1+3 \lambda^{2}} \operatorname{Re}\left(\left(C_{S}-\bar{C}_{S}\right)-\lambda\left(C_{T}-\bar{C}_{T}\right)\right)+\frac{1}{1+3 \lambda^{2}} \operatorname{Re}\left(C_{S} \bar{C}_{S}^{*}-C_{T} \bar{C}_{T}^{*}\right)\right] \\
& \left.+\frac{\vec{\xi}_{e} \cdot\left(\vec{k}_{e} \times \vec{k}_{\nu}\right)}{E_{e} E_{\nu}} \frac{1}{1+3 \lambda^{2}} \operatorname{Im}\left(\left(C_{S}-\bar{C}_{S}\right)-\lambda\left(C_{T}-\bar{C}_{T}\right)\right)\right\}
\end{aligned}
$$

where we have replaced $C_{j}$ and $\bar{C}_{j}$ with $j=V, A$ by $C_{V}=1+\delta C_{V}, \bar{C}_{V}=-1+\delta \bar{C}_{V}, C_{A}=-\lambda+\delta C_{A}$, and $\bar{C}_{A}=\lambda+\delta \bar{C}_{A}$ [1] and neglected also the contributions of the products $\delta C_{j} C_{k}, \delta \bar{C}_{j} C_{k}$ and so on for $j=V, A$ and $k=S, T$. Following 
$[16,17]$ (see also [1]) we have absorbed the contributions the vector and axial vector interactions beyond the SM by the axial coupling constant $\lambda$ and the Cabibbo-Kobayashi-Maskawa matrix element $V_{u d}$.

Thus, the electron-energy and electron-antineutrino angular distribution Eq. (1), taking into account the contributions of interactions beyond the SM, can be transcribed into the form

$$
\begin{aligned}
\frac{d^{3} \lambda_{n}\left(E_{e}, \vec{k}_{e}, \vec{\xi}_{n}, \vec{\xi}_{e}\right)}{d E_{e} d \Omega_{e}}= & \left(1+3 \lambda^{2}\right) \frac{G_{F}^{2}\left|V_{u d}\right|^{2}}{8 \pi^{4}}\left(E_{0}-E_{e}\right)^{2} \sqrt{E_{e}^{2}-m_{e}^{2}} E_{e} F\left(E_{e}, Z=1\right) \zeta^{(\mathrm{SM})}\left(E_{e}\right) \times\left(1+\zeta^{(\mathrm{BSM})}\left(E_{e}\right)\right) \\
& \times\left\{1+b \frac{m_{e}}{E_{e}}+a_{\mathrm{eff}}\left(E_{e}\right) \frac{\vec{k}_{e} \cdot \vec{k}_{\nu}}{E_{e} E_{\nu}}+G_{\mathrm{eff}}\left(E_{e}\right) \frac{\vec{\xi}_{e} \cdot \vec{k}_{e}}{E_{e}}+H_{\mathrm{eff}}\left(E_{e}\right) \frac{\vec{\xi}_{e} \cdot \vec{k}_{\nu}}{E_{\nu}}+K_{\mathrm{e}, \mathrm{eff}}\left(E_{e}\right) \frac{\left(\vec{\xi}_{e} \cdot \vec{k}_{e}\right)\left(\vec{k}_{e} \cdot \vec{k}_{\nu}\right)}{\left(E_{e}+m_{e}\right) E_{e} E_{\nu}}\right. \\
& +L_{\mathrm{eff}}\left(E_{e}\right) \frac{\vec{\xi}_{e} \cdot\left(\vec{k}_{e} \times \vec{k}_{\nu}\right)}{E_{e} E_{\nu}}-3 a_{0} \frac{E_{e}}{M}\left(\frac{\left(\vec{k}_{e} \cdot \vec{k}_{\nu}\right)^{2}}{E_{e}^{2} E_{\nu}^{2}}-\frac{1}{3} \frac{k_{e}^{2}}{E_{e}^{2}}\right)+3 a_{0} \frac{m_{e}}{M}\left(\frac{\left(\vec{\xi}_{e} \cdot \vec{k}_{\nu}\right)\left(\vec{k}_{e} \cdot \vec{k}_{\nu}\right)}{E_{e} E_{\nu}^{2}}-\frac{1}{3} \frac{\vec{\xi}_{e} \cdot \vec{k}_{e}}{E_{e}}\right) \\
& \left.+3 a_{0} \frac{1}{M}\left(\frac{\left(\vec{\xi}_{e} \cdot \vec{k}_{e}\right)\left(\vec{k}_{e} \cdot \vec{k}_{\nu}\right)^{2}}{\left(E_{e}+m_{e}\right) E_{e} E_{\nu}^{2}}-\frac{1}{3}\left(E_{e}-m_{e}\right) \frac{\vec{\xi}_{e} \cdot \vec{k}_{e}}{E_{e}}\right)\right\}
\end{aligned}
$$

where the indices "SM" and "BSM" mean "standard model" and "beyond standard model," respectively. The correlation coefficient $\zeta^{(\mathrm{SM})}\left(E_{e}\right)$ is given in Eq. (25). The Fierz interference term $b$ and the correlation coefficients $X_{\text {eff }}\left(E_{e}\right)$ with $X=a, G, H$ and $K_{e}$ are defined by

$$
\begin{aligned}
b & =\frac{b_{F}}{1+\zeta^{(\mathrm{BSM})}\left(E_{e}\right)}, \quad a_{\mathrm{eff}}\left(E_{e}\right)=\frac{a^{(\mathrm{SM})}\left(E_{e}\right)+a^{(\mathrm{BSM})}\left(E_{e}\right)}{1+\zeta^{(\mathrm{BSM})}\left(E_{e}\right)}, \quad G_{\mathrm{eff}}\left(E_{e}\right)=\frac{G^{(\mathrm{SM})}\left(E_{e}\right)+G^{(\mathrm{BSM})}\left(E_{e}\right)}{1+\zeta^{(\mathrm{BSM})}\left(E_{e}\right)}, \\
H_{\mathrm{e}, \mathrm{eff}}\left(E_{e}\right) & =\frac{H_{e}^{(\mathrm{SM})}\left(E_{e}\right)+H_{e}^{(\mathrm{BSM})}\left(E_{e}\right)}{1+\zeta^{(\mathrm{BSM})}\left(E_{e}\right)}, \quad K_{\mathrm{eff}}\left(E_{e}\right)=\frac{K^{(\mathrm{SM})}\left(E_{e}\right)+K^{(\mathrm{BSM})}\left(E_{e}\right)}{1+\zeta^{(\mathrm{BSM})}\left(E_{e}\right)}, \quad L_{\mathrm{eff}}\left(E_{e}\right)=\frac{L^{(\mathrm{SM})}\left(E_{e}\right)+L^{(\mathrm{BSM})}\left(E_{e}\right)}{1+\zeta^{(\mathrm{BSM})}\left(E_{e}\right)}
\end{aligned}
$$

where the correlation coefficients with index "SM" are adduced in Eqs. (27)-(29). They should be also supplemented by Wilkinson's corrections Eq. (30) and those obtained in [2] (see Chapter III of Ref. [2]). The correlation coefficients $b_{F}$ and the correlation coefficients with index "BSM" are given by

$$
\begin{aligned}
b_{F} & =\frac{1}{1+3 \lambda^{2}} \operatorname{Re}\left(\left(C_{S}-\bar{C}_{S}\right)+3 \lambda\left(C_{T}-\bar{C}_{T}\right)\right), \\
\zeta^{(\mathrm{BSM})}\left(E_{e}\right) & =\frac{1}{2} \frac{1}{1+3 \lambda^{2}}\left(\left|C_{S}\right|^{2}+\left|\bar{C}_{S}\right|^{2}+3\left|C_{T}\right|^{2}+3\left|\bar{C}_{T}\right|^{2}\right), \\
a^{(\mathrm{BSM})}\left(E_{e}\right) & =-\frac{1}{2} \frac{1}{1+3 \lambda^{2}}\left(\left|C_{S}\right|^{2}+\left|\bar{C}_{S}\right|^{2}-\left|C_{T}\right|^{2}-\left|\bar{C}_{T}\right|^{2}\right), \\
G^{(\mathrm{BSM})}\left(E_{e}\right) & =-\frac{1}{1+3 \lambda^{2}} \operatorname{Re}\left(C_{S} \bar{C}_{S}^{*}+3 C_{T} \bar{C}_{T}^{*}\right), \\
H^{(\mathrm{BSM})}\left(E_{e}\right) & =\frac{m_{e}}{E_{e}} \frac{1}{1+3 \lambda^{2}} \operatorname{Re}\left(C_{S} \bar{C}_{S}^{*}-C_{T} \bar{C}_{T}^{*}\right)-\frac{1}{1+3 \lambda^{2}} \operatorname{Re}\left(\left(C_{S}-\bar{C}_{S}\right)-\lambda\left(C_{T}-\bar{C}_{T}\right)\right) \\
K_{e}^{(\mathrm{BSM})}\left(E_{e}\right) & =\frac{1}{1+3 \lambda^{2}} \operatorname{Re}\left(C_{S} \bar{C}_{S}^{*}-C_{T} \bar{C}_{T}^{*}\right)+\frac{1}{1+3 \lambda^{2}} \operatorname{Re}\left(\left(C_{S}-\bar{C}_{S}\right)-\lambda\left(C_{T}-\bar{C}_{T}\right)\right), \\
L_{e}^{(\mathrm{BSM})}\left(E_{e}\right) & =\frac{1}{1+3 \lambda^{2}} \operatorname{Im}\left(\left(C_{S}-\bar{C}_{S}\right)-\lambda\left(C_{T}-\bar{C}_{T}\right)\right) .
\end{aligned}
$$

The correlation coefficient $X_{\text {eff }}\left(E_{e}\right)$ with $X=a, G, H$ and $K_{e}$ are given in the form suitable for the analysis of experimental data of experiments on the searches of interactions beyond the SM [21]. The structure of the correlation coefficients in Eq. (39) agrees well with the structure of corresponding expressions calculated in [11]. The averaged values of the correlation coefficients $X_{\text {eff }}\left(E_{e}\right)$ with $X=a, G, H$, and $K_{e}$ can be obtained with the electron-energy density [3]

$$
\rho_{e}\left(E_{e}\right)=\rho_{e}^{(\mathrm{SM})}\left(E_{e}\right)\left(1+\zeta^{(\mathrm{BSM})}\left(E_{e}\right)\right)=\rho_{e}^{(\mathrm{SM})}\left(E_{e}\right)\left(1+\frac{1}{2} \frac{1}{1+3 \lambda^{2}}\left(\left|C_{S}\right|^{2}+\left|\bar{C}_{S}\right|^{2}+3\left|C_{T}\right|^{2}+3\left|\bar{C}_{T}\right|^{2}\right)\right),
$$

where the electron-energy density $\rho_{e}^{(\mathrm{SM})}\left(E_{e}\right)$ is defined by Eq. (D-59) of Ref. [1]. 


\section{G-ODD CORRELATIONS}

The $G$-parity transformation, i.e., $G=C e^{i \pi I_{2}}$, where $C$ and $I_{2}$ are the charge conjugation and isospin operators, was introduced by Lee and Yang [39] as a symmetry of strong interactions. According to the $G$-transformation properties of hadronic currents, Weinberg divided hadronic currents into two classes, which are $G$-even first class and $G$-odd second class currents [40], respectively. Following Weinberg [40], Gardner and Zhang [19], and Gardner and Plaster [20] the G-odd contribution to the matrix element of the hadronic $n \rightarrow p$ transition in the $V-A$ theory of weak interactions can be taken in the following form

$$
\left\langle p\left(\vec{k}_{p}, \sigma_{p}\right)\left|J_{\mu}^{(+)}(0)\right| n\left(\vec{k}_{n}, \sigma_{n}\right)\right\rangle_{G \text {-odd }}=\bar{u}_{p}\left(\vec{k}_{p}, \sigma_{p}\right)\left(\frac{q_{\mu}}{M} f_{3}(0)+i \frac{1}{M} \sigma_{\mu \nu} \gamma^{5} q^{\nu} g_{2}(0)\right) u_{n}\left(\vec{k}_{n}, \sigma_{n}\right),
$$

where $J_{\mu}^{(+)}(0)=V_{\mu}^{(+)}(0)-A_{\mu}^{(+)}(0), \bar{u}_{p}\left(\vec{k}_{p}, \sigma_{p}\right)$ and $u_{n}\left(\vec{k}_{n}, \sigma_{n}\right)$ are the Dirac wave functions of the proton and neutron [41]; $f_{3}(0)$ and $g_{2}(0)$ are the phenomenological coupling constants defining the strength of the second class currents in the weak decays. The contributions of the second class currents Eq. (41) to the amplitude of the neutron $\beta^{-}$-decay in the nonrelativistic baryon approximation is defined by [3]

$$
\begin{aligned}
M\left(n \rightarrow p e^{-} \bar{\nu}_{e}\right)_{G \text {-odd }}= & -2 m_{n} \frac{G_{F}}{\sqrt{2}} V_{u d}\left\{f_{3}(0) \frac{m_{e}}{M}\left[\varphi_{p}^{\dagger} \varphi_{n}\right]\left[\bar{u}_{e}\left(1-\gamma^{5}\right) v_{\bar{\nu}}\right]+g_{2}(0) \frac{1}{M}\left[\varphi_{p}^{\dagger}\left(\vec{\sigma} \cdot \vec{k}_{p}\right) \varphi_{n}\right]\left[\bar{u}_{e} \gamma^{0}\left(1-\gamma^{5}\right) v_{\bar{\nu}}\right]\right. \\
& \left.-g_{2}(0) \frac{E_{0}}{M}\left[\varphi_{p}^{\dagger} \vec{\sigma} \varphi_{n}\right] \cdot\left[\bar{u}_{e} \vec{\gamma}\left(1-\gamma^{5}\right) v_{\bar{\nu}}\right]\right\},
\end{aligned}
$$

where we have kept only the leading $1 / M$ terms in the large baryon mass expansion. The Hermitian conjugate contribution is

$$
\begin{aligned}
M^{\dagger}\left(n \rightarrow p e^{-} \bar{\nu}_{e}\right)_{G \text {-odd }}= & -2 m_{n} \frac{G_{F}}{\sqrt{2}} V_{u d}\left\{f_{3}^{*}(0) \frac{m_{e}}{M}\left[\varphi_{n}^{\dagger} \varphi_{p}\right]\left[\bar{v}_{\nu}\left(1+\gamma^{5}\right) u_{e}\right]+g_{2}^{*}(0) \frac{1}{M}\left[\varphi_{n}^{\dagger}\left(\vec{\sigma} \cdot \vec{k}_{p}\right) \varphi_{p}\right]\left[\bar{v}_{\nu} \gamma^{0}\left(1-\gamma^{5}\right) u_{e}\right]\right. \\
& \left.-g_{2}^{*}(0) \frac{E_{0}}{M}\left[\varphi_{n}^{\dagger} \vec{\sigma} \varphi_{p}\right] \cdot\left[\bar{v}_{\nu} \vec{\gamma}\left(1-\gamma^{5}\right) u_{e}\right]\right\} .
\end{aligned}
$$

The contributions of the $G$-odd correlations to the squared absolute value of the amplitude of the neutron $\beta^{-}$-decay of polarized electron and unpolarized neutron and proton, summed over polarizations of massive fermions, are equal to

$$
\begin{aligned}
\sum_{\text {pol. }}( & \left.M^{\dagger}\left(n \rightarrow p e^{-} \bar{\nu}_{e}\right) M\left(n \rightarrow p e^{-} \bar{\nu}_{e}\right)_{G \text {-odd }}+M^{\dagger}\left(n \rightarrow p e^{-} \bar{\nu}_{e}\right)_{G \text {-odd }} M\left(n \rightarrow p e^{-} \bar{\nu}_{e}\right)\right) \\
= & 8 m_{n}^{2} G_{F}^{2}\left|V_{u d}\right|^{2}\left\{2 \operatorname{Re} f_{3}(0) \frac{m_{e}}{M}\left[\frac{m_{e}}{E_{e}}+\left(\zeta_{e} \frac{\vec{k}_{e} \cdot \vec{k}_{\nu}}{E_{e} E_{\nu}}-\frac{\vec{\zeta}_{e} \cdot \vec{k}_{\nu}}{E_{\nu}}\right)\right]+2 \operatorname{Im} f_{3}(0) \frac{m_{e}}{M} \frac{\vec{\xi}_{e} \cdot\left(\vec{k}_{e} \times \vec{k}_{\nu}\right)}{E_{e} E_{\nu}}\right. \\
& +2 \lambda \operatorname{Re} g_{2}(0)\left[\frac{1}{M}\left(E_{\nu}+\frac{k_{e}^{2}}{E_{e}}\right)+\frac{E_{e}+E_{\nu}}{M} \frac{\vec{k}_{e} \cdot \vec{k}_{\nu}}{E_{e} E_{\nu}}-\frac{E_{e}+E_{\nu}}{M} \frac{m_{e}}{E_{e}} \zeta_{e}^{0}-\frac{m_{e}}{M} \zeta_{e}^{0} \frac{\vec{k}_{e} \cdot \vec{k}_{\nu}}{E_{e} E_{\nu}}-\frac{m_{e}}{M} \frac{E_{\nu}}{E_{e}} \frac{\vec{\zeta}_{e} \cdot \vec{k}_{\nu}}{E_{\nu}}\right. \\
& \left.\left.+\frac{E_{0}}{M}\left(3-3 \frac{m_{e}}{E_{e}} \zeta_{e}^{0}-\frac{\vec{k}_{e} \cdot \vec{k}_{\nu}}{E_{e} E_{\nu}}+\frac{m_{e}}{E_{e}} \frac{\vec{\zeta}_{e} \cdot \vec{k}_{\nu}}{E_{\nu}}\right)\right]+2 \lambda \operatorname{Im} g_{2}(0) \frac{m_{e}}{M} \frac{\vec{\xi}_{e} \cdot\left(\vec{k}_{e} \times \vec{k}_{\nu}\right)}{E_{e} E_{\nu}}\right\} .
\end{aligned}
$$

For the relative $G$-odd contributions to the correlation coefficients we obtain the following expressions

$$
\begin{aligned}
\frac{\delta \zeta\left(E_{e}\right)_{G \text {-odd }}}{\zeta^{(\mathrm{SM})}\left(E_{e}\right)} & =\frac{2}{1+3 \lambda^{2}} \frac{1}{M}\left\{\operatorname{Re} f_{3}(0) \frac{m_{e}^{2}}{E_{e}}+\lambda \operatorname{Re} g_{2}(0)\left(4 E_{0}-\frac{m_{e}^{2}}{E_{e}}\right)\right\}, \quad \frac{\delta a\left(E_{e}\right)_{G \text {-odd }}}{a^{(\mathrm{SM})}\left(E_{e}\right)}=-\delta \zeta\left(E_{e}\right)_{G \text {-odd }}, \\
\frac{\delta G\left(E_{e}\right)_{G \text {-odd }}}{G^{(\mathrm{SM})}\left(E_{e}\right)} & =\frac{2 \lambda}{1+3 \lambda^{2}} \frac{4 E_{0}}{M} \operatorname{Re} g_{2}(0)-\delta \zeta\left(E_{e}\right)_{G \text {-odd }}, \quad \frac{\delta H\left(E_{e}\right)_{G \text {-odd }}}{H^{(\mathrm{SM})}\left(E_{e}\right)}=\frac{2}{1-\lambda^{2}} \frac{E_{e}}{M}\left(\operatorname{Re} f_{3}(0)-\lambda \operatorname{Re} g_{2}(0)\right)-\delta \zeta\left(E_{e}\right)_{G \text {-odd }}, \\
\frac{\delta K_{e}\left(E_{e}\right)_{G \text {-odd }}}{K_{e}^{(\mathrm{SM})}\left(E_{e}\right)} & =\frac{2}{1-\lambda^{2}} \frac{m_{e}}{M}\left(-\operatorname{Re} f_{3}(0)+\lambda \operatorname{Re} g_{2}(0)\right)-\delta \zeta\left(E_{e}\right)_{G \text {-odd }}, \quad \frac{\delta L\left(E_{e}\right)_{G \text {-odd }}}{L_{e}^{(\mathrm{SM})}\left(E_{e}\right)}=\frac{2}{1-\lambda^{2}} \frac{k_{e}}{\alpha M}\left(\operatorname{Im} f_{3}(0)+\lambda \operatorname{Im} g_{2}(0)\right) .
\end{aligned}
$$


These expressions agree well with the G-odd correlations obtained in [3] and as well as with those by Gardner and Plaster [20]. For $\lambda=-1.2750$ [23] we get

$$
\begin{aligned}
\frac{\delta \zeta\left(E_{e}\right)_{G \text {-odd }}}{\zeta^{(\mathrm{SM})}\left(E_{e}\right)} & =1.85 \times 10^{-4} \operatorname{Re} f_{3}(0) \frac{m_{e}}{E_{e}}+\left(-2.39 \times 10^{-3}+2.36 \times 10^{-4} \frac{m_{e}}{E_{e}}\right) \operatorname{Re} g_{2}(0), \\
\frac{\delta a\left(E_{e}\right)_{G \text {-odd }}}{a^{(\mathrm{SM})}\left(E_{e}\right)} & =-1.85 \times 10^{-4} \operatorname{Re} f_{3}(0) \frac{m_{e}}{E_{e}}+\left(2.39 \times 10^{-3}-2.36 \times 10^{-4} \frac{m_{e}}{E_{e}}\right) \operatorname{Re} g_{2}(0), \\
\frac{\delta G\left(E_{e}\right)_{G \text {-odd }}}{G^{(\mathrm{SM})}\left(E_{e}\right)} & =-1.85 \times 10^{-4} \operatorname{Re} f_{3}(0) \frac{m_{e}}{E_{e}}-2.36 \times 10^{-4} \operatorname{Re} g_{2}(0) \frac{m_{e}}{E_{e}}, \\
\frac{\delta H\left(E_{e}\right)_{G \text {-odd }}}{H^{(\mathrm{SM})}\left(E_{e}\right)} & =\left(-4.40 \times 10^{-3} \frac{E_{e}}{E_{0}}-1.85 \times 10^{-4} \frac{m_{e}}{E_{e}}\right) \operatorname{Re} f_{3}(0)+\left(2.39 \times 10^{-3}-5.61 \times 10^{-3} \frac{E_{e}}{E_{0}}-2.36 \times 10^{-4} \frac{m_{e}}{E_{e}}\right) \operatorname{Re} g_{2}(0), \\
\frac{\delta K_{e}\left(E_{e}\right)_{G \text {-odd }}}{K_{e}^{(\mathrm{SM})}\left(E_{e}\right)} & =\left(1.74 \times 10^{-3}-1.85 \times 10^{-4} \frac{m_{e}}{E_{e}}\right) \operatorname{Re} f_{3}(0)+\left(4.61 \times 10^{-3}-2.36 \times 10^{-4} \frac{m_{e}}{E_{e}}\right) \operatorname{Re} g_{2}(0), \\
\frac{\delta L\left(E_{e}\right)_{G \text {-odd }}}{L^{(\mathrm{SM})}\left(E_{e}\right)} & =\frac{k_{e}}{E_{0}}\left(-0.603 \operatorname{Im} f_{3}(0)+0.769 \operatorname{Im} g_{2}(0)\right) .
\end{aligned}
$$

Following Gardner and Plaster [20] and setting $f_{3}(0)=0$ and $\left|\operatorname{Re} g_{2}(0)\right|<0.01$ we obtain the contributions of the $G$-odd correlations at the level of $10^{-5}$. Of course, the same order of magnitude of the $G$-odd correlations one may get also for $\left|\operatorname{Re} f_{3}(0)\right|<0.01[3]$.

\section{DISCUSSION}

We have analyzed the electron-energy and electronantineutrino angular distribution of the neutron $\beta^{-}$-decay with polarized electron and unpolarized neutron and proton. The correlation coefficients are calculated in the SM to order $10^{-3}$, caused by the weak magnetism and proton recoil of order $O\left(E_{e} / M\right)$ and radiative corrections of order $O(\alpha / \pi)$ Eqs. (27)-(29). The radiative corrections to the correlation coefficients $H\left(E_{e}\right)$ and $K_{e}\left(E_{e}\right)$ are defined by the functions $(\alpha / \pi) h_{n}^{(3)}\left(E_{e}\right)$ and $(\alpha / \pi) h_{n}^{(4)}\left(E_{e}\right)$ [see Eq. (A8) in the Appendix], respectively, which have been never calculated in literature. The correlation coefficients are also supplemented by Wilkinson's higher order corrections Eq. (30) (see also Chapter III of Ref. [2]), which have not been taken in Eqs. (27)-(29) and are induced by (i) the proton recoil in the Coulomb electron-proton finalstate interaction, (ii) the finite proton radius, (iii) the protonlepton convolution, and (iv) the higher-order outer radiative corrections [8].

Taking into account the contribution of interactions beyond the SM we have arrived at the set of correlation coefficients $X_{\text {eff }}\left(E_{e}\right)$ with $X=a, G, H$, and $K_{e}$, given in Eqs. (38) and (39). The structure of these contributions agrees well with the results obtained in [11-14]. These correlation coefficients are presented in the form suitable for the analysis of experimental data on searches of interactions beyond the SM at the level of $10^{-4}$ [21] (see also [1,3]). The analysis of the superallowed $0^{+} \rightarrow$ $0^{+}$transitions, carried out by Hardy and Towner [42] and González-Alonso et al. [43], has shown that in the approximation of real scalar coupling constants such as $C_{S}=-\bar{C}_{S}$, i.e., the neutron and proton couple to righthanded electron and antineutrino, the scalar coupling constants are constrained by $\left|C_{S}\right|=0.0014(13)$ and $\left|C_{S}\right|=0.0014(12)$. Such a small value of the scalar coupling constants commensurable with zero can be justified by the property of the scalar density $\bar{\psi}_{p} \psi_{n}$ with respect to the $G$-transformation $[39,40]$ (see also $[41,44]$ ). Indeed, the scalar density $\bar{\psi}_{p} \psi_{n}=\bar{\psi}_{N} \tau^{(+)} \psi_{N}$, where $\psi_{N}$ is the field operator of the nucleon isospin doublet with components $\left(\psi_{p}, \psi_{n}\right)$ and $\tau^{(+)}=\left(\tau^{1}+i \tau^{2}\right) / 2$ is the isospin $2 \times 2$ Pauli matrix such as $\vec{\tau}=\left(\tau^{1}, \tau^{2}, \tau^{3}\right)$ [32], is $G$-odd $[41,44]$. According to Weinberg [40], the contributions of $G$-odd hadronic currents or second class hadronic currents to the weak decays are suppressed with respect to the contributions of $G$-even or first class hadronic currents. As a result one may expect that in the neutron $\beta^{-}$-decays the contributions of the tensor density $\bar{\psi}_{p} \sigma_{\mu \nu} \psi_{n}=\bar{\psi}_{N} \sigma_{\mu \nu} \tau^{(+)} \psi_{N}$, which is $G$-even $[41,44]$, should be larger than the contribution of the scalar density $\bar{\psi}_{p} \psi_{n}=\bar{\psi}_{N} \tau^{(+)} \psi_{N}$, which is $G$-odd [41,44]. These estimates agree well with the contributions of order $10^{-5}$ of $G$-odd terms in the matrix element of the hadronic $n \rightarrow p$ transition to the correlation coefficients, which we have calculated in Sec. X in agreement with the results obtained by Gardner and Plaster [20] and Ivanov et al. [3].

It is obvious that the analysis of experimental data of experiments on the searches of contributions of interactions beyond the SM at the level of $10^{-4}$ or even better [21] demands a robust SM theoretical background with 
corrections at the level of $10^{-5}$. These are (i) Wilkinson's corrections [2] and (ii) corrections of order $O\left(E_{e}^{2} / M^{2}\right)$ defined by the weak magnetism and proton recoil, calculated to next-to-next-to-leading order in the large nucleon mass expansion, the radiative corrections of order $O\left(\alpha E_{e} / M\right)$, calculated to next-to-leading order in the large nucleon mass expansion, and the radiative corrections of order $O\left(\alpha^{2} / \pi^{2}\right)$, calculated to leading order in the large nucleon mass expansion [22]. These theoretical corrections should provide for the analysis of experimental data of "discovery" experiments the required $5 \sigma$ level of experimental uncertainties of a few parts in $10^{-5}$ [2]. An important role of strong low-energy interactions for a correct gauge invariant calculation of radiative corrections of order $O\left(\alpha E_{e} / M\right)$ and $O\left(\alpha^{2} / \pi^{2}\right)$ as functions of the electron energy $E_{e}$ has been pointed out in [22]. This agrees with Weinberg's assertion about important role of strong low-energy interactions in decay processes [45]. A procedure for the calculation of these radiative corrections to the neutron $\beta^{-}$-decays with a consistent account for contributions of strong low-energy interactions, leading to gauge invariant observable expressions dependent on the electron energy $E_{e}$ determined at the confidence level of Sirlin's radiative corrections [5], has been proposed in [22]. As we have shown that the contributions of the $G$-odd correlations are at the level of $10^{-5}$. Hence, the SM corrections of order $10^{-5}$ should be important also as a theoretical background for the analysis of experimental data on the search of the contributions of the $G$-odd correlations in the neutron $\beta^{-}$-decays.

Finally, we would like to make some comments on the radiative corrections of order $O(\alpha / \pi)$, which we have calculated to the correlation coefficients of the electronenergy and electron-antineutrino angular distribution of the neutron $\beta^{-}$-decay with polarized electron and unpolarized neutron and proton. Such a calculation has been performed in analogy to the calculation of radiative corrections to the neutron lifetime and the correlation coefficients of the electron-energy and electron-antineutrino angular distributions of the neutron $\beta^{-}$-decay with polarized neutron and unpolarized proton and electron, carried out by Sirlin [5] and Shann [6] (see also [1,7]), and of the neutron $\beta^{-}$-decay with polarized neutron and electron and unpolarized proton [2]. The radiative corrections to the correlation coefficients define the level of accuracy of the theoretical background for the measurements of these correlation coefficients. However, as has been pointed out by Glück [46], these results may not be applicable to precise analyses of recoil measurements, dealing with recoil energy and angular distributions. For the neutron $\beta^{-}$-decay with polarized neutron and unpolarized proton and electron the radiative corrections to the proton recoil-energy and angular distribution have been calculated in [31] (see also [7]). The calculation of radiative corrections to the electron-proton recoil-energy and angular distribution for the neutron $\beta^{-}$decay with polarized electron and unpolarized neutron and proton demands a special consideration (see, e.g., [31]) and goes beyond the scope of this paper. We are planning to perform such a calculation in our forthcoming publication.

\section{ACKNOWLEDGMENTS}

We thank Hartmut Abele for stimulating discussions. The work of A. N. I. was supported by the Austrian "Fonds zur Förderung der Wissenschaftlichen Forschung" (FWF) under Contracts No. P31702-N27, No. P26781-N20 and No. P26636-N20 and "Deutsche Förderungsgemeinschaft" (DFG) AB 128/5-2. The work of R. Höllwieser was supported by the Deutsche Forschungsgemeinschaft in the SFB/TR 55. The work of M. Wellenzohn was supported by the MA 23 (FH-Call 16) under the project "PhotonikStiftungsprofessur für Lehre".

\section{APPENDIX: NEUTRON RADIATIVE $\beta^{-}$-DECAY WITH POLARIZED ELECTRON AND UNPOLARIZED NEUTRON, PROTON, AND PHOTON}

In this Appendix we calculate the electron-energy and angular distribution of the rate of the neutron radiative $\beta^{-}$decay $n \rightarrow p+e^{-}+\bar{\nu}_{e}+\gamma$ with polarized electron and unpolarized neutron, proton, and photon. Such a distribution is important for the cancellation of infrared divergences in the neutron lifetime and correlation coefficients of the neutron $\beta^{-}$-decay $[1,2,5]$. The Feynman diagrams of the neutron radiative $\beta^{-}$-decay are shown in Fig. 2.

Following [1,2] (see also [22,31]) the energy and angular distribution of the neutron radiative $\beta^{-}$-decay with polarized electron and unpolarized neutron and proton is

$$
\begin{aligned}
& \frac{d^{8} \lambda_{\beta_{c} \gamma}\left(E_{e}, \vec{k}_{e}, \vec{\xi}_{e}, \vec{k}_{\nu}, \vec{q}\right)_{\lambda \lambda^{\prime}}}{d \omega d E_{e} d \Omega_{e} d \Omega_{\nu} d \Omega_{\gamma}} \\
& =\frac{\alpha}{2 \pi}\left(1+3 \lambda^{2}\right) \frac{G_{F}^{2}\left|V_{u d}\right|^{2}}{(2 \pi)^{6}} \sqrt{E_{e}^{2}-m_{e}^{2}} F\left(E_{e}, Z=1\right) \frac{\left(E_{0}-E_{e}-\omega\right)^{2}}{\left(E_{e}-\vec{n}_{\vec{q}} \cdot \vec{k}_{e}\right)^{2}} \frac{1}{\omega} \\
& \quad \times \frac{1}{16}\left\{\operatorname{tr}\left\{\left(\hat{k}_{e}+m_{e} \gamma^{5} \hat{\zeta}_{e}\right) Q_{\lambda} \gamma^{0} \bar{Q}_{\lambda^{\prime}}\left(1-\gamma^{5}\right)\right\}+a_{0} \frac{\vec{k}_{\nu}}{E_{\nu}} \cdot \operatorname{tr}\left\{\left(\hat{k}_{e}+m_{e} \gamma^{5} \hat{\zeta}_{e}\right) Q_{\lambda} \vec{\gamma} \bar{Q}_{\lambda^{\prime}}\left(1-\gamma^{5}\right)\right\}\right\},
\end{aligned}
$$


where $d \Omega_{e}, d \Omega_{\nu}$, and $d \Omega_{\gamma}$ are elements of the solid angels of the electron, antineutrino, and photon, respectively. Then, $Q_{\lambda}=2 \varepsilon_{\lambda}^{*}(q) \cdot k_{e}+\hat{\varepsilon}_{\lambda}^{*}(q) \hat{q}$ and $\bar{Q}_{\lambda^{\prime}}=\gamma^{0} Q_{\lambda^{\prime}}^{\dagger} \gamma^{0}=2 \varepsilon_{\lambda^{\prime}}(q) \cdot k_{e}+\hat{q} \hat{\varepsilon}_{\lambda^{\prime}}(q)$, where $\varepsilon_{\lambda}^{*}(q)$ (or $\left.\varepsilon_{\lambda^{\prime}}(q)\right)$ and $q=(\omega, \vec{q})=\left(\omega, \omega \vec{n}_{\vec{q}}\right)$ are the polarization vector and 4-momentum of the photon obeying the constraints $\varepsilon_{\lambda}^{*}(q) \cdot q=0$ (or $\left.\varepsilon_{\lambda^{\prime}}(q) \cdot q=0\right)$ and $q^{2}=0, \vec{n}_{\vec{q}}=\vec{q} / \omega$ is a unit vector and $\lambda\left(\lambda^{\prime}\right)=1,2$ defines physical polarization states of the photon. In Eq. (A1) the traces over Dirac matrices in the covariant form are defined by

$$
\begin{aligned}
\frac{1}{16} \operatorname{tr}\{ & \left.\hat{a} Q_{\lambda} \gamma^{\mu} \bar{Q}_{\lambda^{\prime}}\left(1-\gamma^{5}\right)\right\}=\left(\varepsilon_{\lambda}^{*} \cdot k_{e}\right)\left(\varepsilon_{\lambda^{\prime}} \cdot k_{e}\right) a^{\mu}+\frac{1}{2}\left(\left(\varepsilon_{\lambda}^{*} \cdot k_{e}\right)\left(\varepsilon_{\lambda^{\prime}} \cdot a\right)+\left(\varepsilon_{\lambda}^{*} \cdot a\right)\left(\varepsilon_{\lambda^{\prime}} \cdot k_{e}\right)-\left(\varepsilon_{\lambda}^{*} \cdot \varepsilon_{\lambda}^{*}\right)(a \cdot q)\right) q^{\mu} \\
& -\frac{1}{2}\left(\left(\varepsilon_{\lambda}^{*} \cdot k_{e}\right) \varepsilon_{\lambda^{\prime}}^{\mu}+\varepsilon_{\lambda}^{* \mu}\left(\varepsilon_{\lambda^{\prime}} \cdot k_{e}\right)\right)(a \cdot q)-\frac{1}{2} i \varepsilon^{\mu \nu \alpha \beta}\left(\left(\varepsilon_{\lambda}^{*} \cdot k_{e}\right) \varepsilon_{\lambda^{\prime} \nu}-\varepsilon_{\lambda \nu}^{*}\left(\varepsilon_{\lambda^{\prime}} \cdot k_{e}\right)\right) a_{\alpha} q_{\beta}-\frac{1}{2} i q^{\mu} \varepsilon^{\rho \varphi \alpha \beta} \varepsilon_{\lambda \rho}^{*} \varepsilon_{\lambda^{\prime} \varphi} a_{\alpha} q_{\beta},
\end{aligned}
$$

where $a=k_{e}$ and $a=m_{e} \zeta_{e}$, and $\varepsilon^{\alpha \nu \mu \beta}$ is the Levi-Civita tensor defined by $\varepsilon^{0123}=1$ and $\varepsilon_{\alpha \nu \mu \beta}=-\varepsilon^{\alpha \nu \mu \beta}$ [32]. Plugging Eq. (A2) into Eq. (A1), using the Coulomb gauge [1,2] (see also [22,31]) and summing over photon polarizations we obtain the following expression for the energy and angular distribution of the neutron radiative $\beta^{-}$-decay

$$
\begin{aligned}
& \frac{d^{8} \lambda_{\beta_{c}^{-\gamma}}\left(E_{e}, \vec{k}_{e}, \vec{\xi}_{e}, \vec{k}_{\nu}, \vec{q}\right)}{d \omega d E_{e} d \Omega_{e} d \Omega_{\nu} d \Omega_{\gamma}} \\
& =\frac{\alpha}{2 \pi}\left(1+3 \lambda^{2}\right) \frac{G_{F}^{2}\left|V_{u d}\right|^{2}}{(2 \pi)^{6}} \sqrt{E_{e}^{2}-m_{e}^{2}} E_{e} F\left(E_{e}, Z=1\right)\left(E_{0}-E_{e}-\omega\right)^{2} \frac{1}{\omega}\left\{\left[\frac{\beta^{2}-\left(\vec{n}_{\vec{q}} \cdot \vec{\beta}\right)^{2}}{\left(1-\vec{n}_{\vec{q}} \cdot \vec{\beta}\right)^{2}}\left(1+\frac{\omega}{E_{e}}\right)+\frac{1}{1-\vec{n}_{\vec{q}} \cdot \vec{\beta}} \frac{\omega^{2}}{E_{e}^{2}}\right]\right. \\
& \quad+a_{0} \frac{\vec{k}_{\nu}}{E_{\nu}} \cdot\left[\vec{\beta}\left(\frac{\beta^{2}-\left(\vec{n}_{\vec{q}} \cdot \vec{\beta}\right)^{2}}{\left(1-\vec{n}_{\vec{q}} \cdot \vec{\beta}\right)^{2}}+\frac{1}{1-\vec{n}_{\vec{q}} \cdot \vec{\beta}} \frac{\omega}{E_{e}}\right)+\vec{n}_{\vec{q}}\left(-\frac{m_{e}^{2}}{E_{e}^{2}} \frac{1}{\left(1-\vec{n}_{\vec{q}} \cdot \vec{\beta}\right)^{2}} \frac{\omega}{E_{e}}+\frac{1}{1-\vec{n}_{\vec{q}} \cdot \vec{\beta}} \frac{\omega}{E_{e}}+\frac{1}{1-\vec{n}_{\vec{q}} \cdot \vec{\beta}} \frac{\omega^{2}}{E_{e}^{2}}\right)\right] \\
& \quad+\frac{\vec{\xi}_{e} \cdot \vec{k}_{e}}{E_{e}}\left[-\frac{\beta^{2}-\left(\vec{n}_{\vec{q}} \cdot \vec{\beta}\right)^{2}}{\left(1-\vec{n}_{\vec{q}} \cdot \vec{\beta}\right)^{2}}-\frac{1}{\left(1-\vec{n}_{\vec{q}} \cdot \vec{\beta}\right)^{2}} \frac{\omega}{E_{e}}-\frac{1}{\left(1-\vec{n}_{\vec{q}} \cdot \vec{\beta}\right)^{2}} \frac{\omega^{2}}{E_{e}^{2}}\right]+\frac{m_{e}}{E_{e}}\left[\left(-\frac{\vec{n}_{\vec{q}} \cdot \vec{\zeta}_{e}}{1-\vec{n}_{\vec{q}} \cdot \vec{\beta}}+\frac{\vec{n}_{\vec{q}} \cdot \vec{\zeta}_{e}}{\left(1-\vec{n}_{\vec{q}} \cdot \vec{\beta}\right)^{2}}\right) \frac{\omega}{E_{e}}\right. \\
& \left.\quad+\frac{\vec{n}_{\vec{q}} \cdot \vec{\zeta}_{e}}{\left(1-\vec{n}_{\vec{q}} \cdot \vec{\beta}\right)^{2}} \frac{\omega^{2}}{E_{e}^{2}}\right]-a_{0} \frac{m_{e}}{E_{e}} \frac{\vec{k}_{\nu}}{E_{\nu}} \cdot\left[\vec{\zeta}_{e} \frac{\beta^{2}-\left(\vec{n}_{\vec{q}} \cdot \vec{\beta}\right)^{2}}{\left(1-\vec{n}_{\vec{q}} \cdot \vec{\beta}\right)^{2}}+\vec{\beta} \frac{\zeta_{e}^{0}-\vec{n}_{\vec{q}} \cdot \vec{\zeta}_{e}}{\left(1-\vec{n}_{\vec{q}} \cdot \vec{\beta}\right)^{2}} \frac{\omega}{E_{e}}+\vec{n}_{\vec{q}}\left[\frac{\zeta_{e}^{0}}{1-\vec{n}_{\vec{q}} \cdot \vec{\beta}} \frac{\omega}{E_{e}}+\frac{\zeta_{e}^{0}-\vec{n}_{\vec{q}} \cdot \vec{\zeta}_{e}}{\left(1-\vec{n}_{\vec{q}} \cdot \vec{\beta}\right)^{2}} \frac{\omega^{2}}{E_{e}^{2}}\right]\right\} .
\end{aligned}
$$

The integration over directions of the photon momentum we carry out using the results obtain in the Appendix of Ref. [2]. As result the energy and angular distribution Eq. (A3) takes the form

$$
\begin{aligned}
\frac{d^{6} \lambda_{\beta_{c}^{-} \gamma}\left(E_{e}, \vec{k}_{e}, \vec{\xi}_{e}, \vec{k}_{\nu}, \vec{q}\right)}{d \omega d E_{e} d \Omega_{e} d \Omega_{\nu}} & =\frac{\alpha}{\pi}\left(1+3 \lambda^{2}\right) \frac{G_{F}^{2}\left|V_{u d}\right|^{2}}{(2 \pi)^{5}} \sqrt{E_{e}^{2}-m_{e}^{2}} E_{e} F\left(E_{e}, Z=1\right)\left(E_{0}-E_{e}-\omega\right)^{2} \frac{1}{\omega} \\
& \times\left\{\left\{\left(1+\frac{\omega}{E_{e}}+\frac{1}{2} \frac{\omega^{2}}{E_{e}^{2}}\right)\left[\frac{1}{\beta} \ln \left(\frac{1+\beta}{1-\beta}\right)-2\right]+\frac{\omega^{2}}{E_{e}^{2}}\right\}+a_{0} \frac{\vec{k}_{e} \cdot \vec{k}_{\nu}}{E_{e} E_{\nu}}\left[1+\frac{1}{\beta^{2}} \frac{\omega}{E_{e}}\left(1+\frac{1}{2} \frac{\omega}{E_{e}}\right)\right]\left[\frac{1}{\beta} \ell n\left(\frac{1+\beta}{1-\beta}\right)-2\right]\right. \\
& -\frac{\vec{\xi}_{e} \cdot \vec{k}_{e}}{E_{e}}\left[1+\frac{1}{\beta^{2}} \frac{\omega}{E_{e}}\left(1+\frac{1}{2} \frac{\omega}{E_{e}}\right)\right]\left[\frac{1}{\beta} \ln \left(\frac{1+\beta}{1-\beta}\right)-2\right]-\frac{\vec{\xi}_{e} \cdot \vec{k}_{\nu}}{E_{\nu}} a_{0} \frac{m_{e}}{E_{e}}\left[\frac{1}{\beta} \ell n\left(\frac{1+\beta}{1-\beta}\right)-2\right]\left(1-\frac{1}{2 \beta^{2}} \frac{\omega^{2}}{E_{e}^{2}}\right) \\
& -a_{0} \frac{\left(\vec{\xi}_{e} \cdot \vec{k}_{e}\right)\left(\vec{k}_{e} \cdot \vec{k}_{\nu}\right)}{\left(E_{e}+m_{e}\right) E_{e} E_{\nu}}\left\{\left[\frac{1}{\beta} \ell n\left(\frac{1+\beta}{1-\beta}\right)-2\right]\left(1-\frac{1}{2 \beta^{2}} \frac{\omega^{2}}{E_{e}^{2}}\right)+\left(1+\sqrt{1-\beta^{2}}\right)\left[\frac{1}{1-\beta^{2}} \frac{\omega}{E_{e}}+\frac{1}{2 \beta^{2}}\left[\frac{1}{\beta} \ell n\left(\frac{1+\beta}{1-\beta}\right)-2\right]\right.\right. \\
& \left.\left.\times \frac{\omega}{E_{e}}+\frac{1}{2 \beta^{2}}\left[\frac{1}{\beta} \ell n\left(\frac{1+\beta}{1-\beta}\right)-\frac{2}{1-\beta^{2}}\right] \frac{\omega}{E_{e}}\left(1-\frac{\omega}{E_{e}}\right)+\frac{1}{4 \beta^{4}}\left[\frac{3}{\beta} \ell n\left(\frac{1+\beta}{1-\beta}\right)-4-\frac{2}{1-\beta^{2}}\right] \frac{\omega^{2}}{E_{e}^{2}}\right\}\right\} .
\end{aligned}
$$

The first three correlation coefficients agree well with the results, obtained in [1] (see Eq. (B-11) of Ref. [1]) and [2] (see Eq. (A-5) of Ref. [2]). Having integrated over the photon energy in the region $\omega_{\min } \leq \omega \leq E_{0}-E_{e}$, where $\omega_{\text {in }}$ is an infrared cutoff [1], we arrive at the expression 


$$
\begin{aligned}
& \frac{d^{5} \lambda_{\beta_{c}^{-} \gamma}\left(E_{e}, \vec{k}_{e}, \vec{\xi}_{e}, \vec{k}_{\nu}\right)}{d E_{e} d \Omega_{e} d \Omega_{\nu}} \\
& =\frac{\alpha}{\pi}\left(1+3 \lambda^{2}\right) \frac{G_{F}^{2}\left|V_{u d}\right|^{2}}{(2 \pi)^{5}} \sqrt{E_{e}^{2}-m_{e}^{2}} E_{e} F\left(E_{e}, Z=1\right)\left(E_{0}-E_{e}\right)^{2}\left\{g_{\beta_{c}^{-} \gamma}^{(1)}\left(E_{e}, \omega_{\min }\right)+\frac{\vec{k}_{e} \cdot \vec{k}_{\nu}}{E_{e} E_{\nu}} a_{0} g_{\beta_{c} \gamma}^{(2)}\left(E_{e}, \omega_{\min }\right)\right. \\
& \left.\quad-\frac{\vec{\xi}_{e} \cdot \vec{k}_{e}}{E_{e}} g_{\beta_{c}^{-} \gamma}^{(2)}\left(E_{e}, \omega_{\min }\right)-\frac{\vec{\xi}_{e} \cdot \vec{k}_{\nu}}{E_{\nu}} a_{0} \frac{m_{e}}{E_{e}} g_{\beta_{c}^{-} \gamma}^{(5)}\left(E_{e}, \omega_{\min }\right)-a_{0} \frac{\left(\vec{\xi}_{e} \cdot \vec{k}_{e}\right)\left(\vec{k}_{e} \cdot \vec{k}_{\nu}\right)}{\left(E_{e}+m_{e}\right) E_{e} E_{\nu}} g_{\beta_{c}^{-} \gamma}^{(6)}\left(E_{e}, \omega_{\min }\right)\right\}
\end{aligned}
$$

The functions $g_{\beta_{c}^{-\gamma}}^{(1)}\left(E_{e}, \omega_{\min }\right)$ and $g_{\beta_{c} \gamma}^{(2)}\left(E_{e}, \omega_{\min }\right)$ have been calculated in [1,2], whereas the functions $g_{\beta_{c}^{-} \gamma}^{(5)}\left(E_{e}, \omega_{\min }\right)$ and $g_{\beta_{c}^{-} \gamma}^{(6)}\left(E_{e}, \omega_{\min }\right)$ are defined by the integrals

$$
\begin{aligned}
g_{\beta_{c}^{-} \gamma}^{(5)}\left(E_{e}, \omega_{\min }\right)= & \int_{\omega_{\min }}^{E_{0}-E_{e}} \frac{d \omega}{\omega} \frac{\left(E_{0}-E_{e}-\omega\right)^{2}}{\left(E_{0}-E_{e}\right)^{2}}\left[\frac{1}{\beta} \ln \left(\frac{1+\beta}{1-\beta}\right)-2\right]\left(1-\frac{1}{2 \beta^{2}} \frac{\omega^{2}}{E_{e}^{2}}\right), \\
g_{\beta_{c}^{-} \gamma}^{(6)}\left(E_{e}, \omega_{\min }\right)= & \int_{\omega_{\min }}^{E_{0}-E_{e}} \frac{d \omega}{\omega} \frac{\left(E_{0}-E_{e}-\omega\right)^{2}}{\left(E_{0}-E_{e}\right)^{2}}\left\{\left\{\left[\frac{1}{\beta} \ln \left(\frac{1+\beta}{1-\beta}\right)-2\right]\left(1-\frac{1}{2 \beta^{2}} \frac{\omega^{2}}{E_{e}^{2}}\right)\right.\right. \\
& +\left(1+\sqrt{1-\beta^{2}}\right)\left\{\left[\frac{1}{1-\beta^{2}} \frac{\omega}{E_{e}}+\frac{1}{2 \beta^{2}}\left[\frac{1}{\beta} \ln \left(\frac{1+\beta}{1-\beta}\right)-2\right] \frac{\omega}{E_{e}}\right.\right. \\
& \left.\left.+\frac{1}{2 \beta^{2}}\left[\frac{1}{\beta} \ln \left(\frac{1+\beta}{1-\beta}\right)-\frac{2}{1-\beta^{2}}\right] \frac{\omega}{E_{e}}\left(1-\frac{\omega}{E_{e}}\right)+\frac{1}{4 \beta^{4}}\left[\frac{3}{\beta} \ln \left(\frac{1+\beta}{1-\beta}\right)-4-\frac{2}{1-\beta^{2}}\right] \frac{\omega^{2}}{E_{e}^{2}}\right\}\right\} .
\end{aligned}
$$

The results of the integration are equal to

$$
\begin{aligned}
g_{\beta_{c}^{-} \gamma}^{(5)}\left(E_{e}, \omega_{\min }\right)= & {\left[\ln \left(\frac{E_{0}-E_{e}}{\omega_{\min }}\right)-\frac{3}{2}-\frac{1}{24 \beta^{2}} \frac{\left(E_{0}-E_{e}\right)^{2}}{E_{e}^{2}}\right]\left[\frac{1}{\beta} \ln \left(\frac{1+\beta}{1-\beta}\right)-2\right], } \\
g_{\beta_{c}^{-} \gamma}^{(6)}\left(E_{e}, \omega_{\min }\right)= & {\left[\ln \left(\frac{E_{0}-E_{e}}{\omega_{\min }}\right)-\frac{3}{2}-\frac{1}{24 \beta^{2}} \frac{\left(E_{0}-E_{e}\right)^{2}}{E_{e}^{2}}\right]\left[\frac{1}{\beta} \ln \left(\frac{1+\beta}{1-\beta}\right)-2\right]+\left(1+\sqrt{1-\beta^{2}}\right) \frac{1}{3} \frac{\left(E_{0}-E_{e}\right)}{E_{e}} } \\
& \times\left\{\frac{1}{1-\beta^{2}}+\frac{1}{2 \beta^{2}}\left[\frac{1}{\beta} \ln \left(\frac{1+\beta}{1-\beta}\right)-2\right]+\frac{1}{2 \beta^{2}}\left[\frac{1}{\beta} \ln \left(\frac{1+\beta}{1-\beta}\right)-\frac{2}{1-\beta^{2}}\right]\left(1-\frac{1}{4} \frac{E_{0}-E_{e}}{E_{e}}\right)\right. \\
& \left.+\frac{1}{16 \beta^{4}}\left[\frac{3}{\beta} \ln \left(\frac{1+\beta}{1-\beta}\right)-4-\frac{2}{1-\beta^{2}}\right] \frac{E_{0}-E_{e}}{E_{e}}\right\} .
\end{aligned}
$$

Now we are able to define the electron-energy and electron-antineutrino angular distribution for the neutron $\beta^{-}$-decay with polarized electron and unpolarized neutron and proton, where the correlation coefficients are calculated to order $10^{-3}$, caused by the weak magnetism and proton recoil of order $O\left(E_{e} / M\right)$ and radiative corrections of order $O(\alpha / \pi)$.

The radiative corrections of order $O(\alpha / \pi)$ to the correlation coefficients of the neutron $\beta^{-}$-decay with polarized electron and unpolarized neutron and proton are defined by the function $g_{n}\left(E_{e}\right)$ and the functions

$$
\begin{aligned}
f_{n}\left(E_{e}\right)= & \lim _{\omega_{\min } \rightarrow 0}\left[g_{\beta_{c}^{-\gamma} \gamma}^{(2)}\left(E_{e}, \omega_{\min }\right)-g_{\beta_{c}^{-} \gamma}^{(1)}\left(E_{e}, \omega_{\min }\right)\right]+g_{F}\left(E_{e}\right) \frac{m_{e}}{E_{e}}=\frac{1}{3} \frac{1-\beta^{2}}{\beta^{2}} \frac{E_{0}-E_{e}}{E_{e}}\left(1+\frac{1}{8} \frac{E_{0}-E_{e}}{E_{e}}\right) \\
& \times\left[\frac{1}{\beta} \ell n\left(\frac{1+\beta}{1-\beta}\right)-2\right]-\frac{1}{12} \frac{\left(E_{0}-E_{e}\right)^{2}}{E_{e}^{2}}+\frac{1-\beta^{2}}{2 \beta} \ell n\left(\frac{1+\beta}{1-\beta}\right), \\
h_{n}^{(3)}\left(E_{e}\right)= & \lim _{\omega_{\min } \rightarrow 0}\left[g_{\beta_{c}^{\prime} \gamma}^{(5)}\left(E_{e}, \omega_{\min }\right)-g_{\beta_{c}^{-} \gamma}^{(1)}\left(E_{e}, \omega_{\min }\right)\right]+g_{F}\left(E_{e}\right) \frac{m_{e}}{E_{e}}-g_{F}\left(E_{e}\right) \frac{E_{e}}{m_{e}} \\
= & \left(-3+\frac{1}{3} \frac{E_{0}-E_{e}}{E_{e}}-\frac{1-\beta^{2}}{24 \beta^{2}} \frac{\left(E_{0}-E_{e}\right)^{2}}{E_{e}^{2}}\right)\left[\frac{1}{\beta} \ell n\left(\frac{1+\beta}{1-\beta}\right)-2\right]-\frac{\beta}{2} \ell n\left(\frac{1+\beta}{1-\beta}\right),
\end{aligned}
$$




$$
\begin{aligned}
h_{n}^{(4)}\left(E_{e}\right)= & \lim _{\omega_{\min } \rightarrow 0}\left[g_{\beta_{c}^{-} \gamma}^{(6)}\left(E_{e}, \omega_{\min }\right)-g_{\beta_{c}^{-} \gamma}^{(1)}\left(E_{e}, \omega_{\min }\right)\right]+g_{F}\left(E_{e}\right) \frac{m_{e}}{E_{e}}-g_{F}\left(E_{e}\right) \frac{E_{e}}{m_{e}} \\
= & \left(-3+\frac{1}{3} \frac{E_{0}-E_{e}}{E_{e}}-\frac{1-\beta^{2}}{24 \beta^{2}} \frac{\left(E_{0}-E_{e}\right)^{2}}{E_{e}^{2}}\right)\left[\frac{1}{\beta} \ln \left(\frac{1+\beta}{1-\beta}\right)-2\right]-\frac{\beta}{2} \ell n\left(\frac{1+\beta}{1-\beta}\right)+\left(1+\sqrt{1-\beta^{2}}\right) \frac{1}{3} \frac{\left(E_{0}-E_{e}\right)}{E_{e}} \\
& \times\left\{\frac{1}{1-\beta^{2}}+\frac{1}{2 \beta^{2}}\left[\frac{1}{\beta} \ell n\left(\frac{1+\beta}{1-\beta}\right)-2\right]+\frac{1}{2 \beta^{2}}\left[\frac{1}{\beta} \ln \left(\frac{1+\beta}{1-\beta}\right)-\frac{2}{1-\beta^{2}}\right]\left(1-\frac{1}{4} \frac{E_{0}-E_{e}}{E_{e}}\right)\right. \\
& \left.+\frac{1}{16 \beta^{4}}\left[\frac{3}{\beta} \ell n\left(\frac{1+\beta}{1-\beta}\right)-4-\frac{2}{1-\beta^{2}}\right] \frac{E_{0}-E_{e}}{E_{e}}\right\} .
\end{aligned}
$$

For the calculation of the radiative corrections to the neutron lifetime and correlation coefficients of the neutron $\beta^{-}$-decay the integral

$$
J\left(\beta, \kappa_{\mathrm{IR}}\right)=\int \frac{d \omega}{\omega} \int \frac{d \Omega_{\gamma}}{4 \pi} \frac{\beta^{2}-\left(\vec{n}_{\vec{q}} \cdot \vec{\beta}\right)^{2}}{\left(1-\vec{n}_{\vec{q}} \cdot \vec{\beta}\right)^{2}},
$$

which is logarithmically divergent in the infrared region of photon energy [5], plays an important role. As has been pointed out in [1], the result of the calculation of this integral depends on the regularization procedure, where $\kappa_{\mathrm{IR}}$ is an infrared parameter.

Using the infrared cutoff regularization $\kappa_{\mathrm{IR}}=\omega_{\min } \leq$ $\omega \leq\left(E_{0}-E_{e}\right)$, where $\omega_{\min }$ may be also treated as a photon-energy threshold of the detector, we get
$J\left(\beta, \omega_{\min }\right)=\ln \left(\frac{E_{0}-E_{e}}{\omega_{\min }}\right)\left[\frac{1}{\beta} \ln \left(\frac{1+\beta}{1-\beta}\right)-2\right]$.

In turn, the use of the finite photon-mass $\mu$ (FPM) regularization

$$
J(\beta, \mu)=\int \frac{d^{3} q}{4 \pi q_{0}^{3}} \frac{\beta^{2}-(\vec{v} \cdot \vec{\beta})^{2}}{(1-\vec{v} \cdot \vec{\beta})^{2}},
$$

where $q_{0}=\sqrt{\omega^{2}+\mu^{2}}$ and $\vec{v}=\vec{q} / q_{0}$ are energy and velocity of a photon with mass $\mu$, gives one (see Eq. (B-26) of Ref. [1])

$$
J(\beta, \mu)=\ell n\left(\frac{2\left(E_{0}-E_{e}\right.}{\mu}\right)\left[\frac{1}{\beta} \ell n\left(\frac{1+\beta}{1-\beta}\right)-2\right]+1+\frac{1}{\beta} \ln \left(\frac{1+\beta}{1-\beta}\right)-\frac{1}{\beta} \ell n^{2}\left(\frac{1+\beta}{1-\beta}\right)-\frac{1}{2} \operatorname{Li}_{2}\left(\frac{2 \beta}{1+\beta}\right),
$$

where $\operatorname{Li}_{2}(x)$ is a Polylogarithmic function $[47,48]$. We would like to emphasize that the infinitesimal photon mass $\mu$, providing a Lorentz covariant regularization of infrared divergences in the neutron $\beta^{-}$-decays, cannot be identified with the infrared cutoff $\omega_{\min }$, which can be treated as a photon-energy threshold of the detector [49-51] (see also [1]). Nevertheless, the use of the Lorentz covariant FPM regularization is important only for the calculation of the function $g_{n}\left(E_{e}\right)$, defining the radiative corrections to the neutron lifetime [5]. It is required by gauge invariance of radiative corrections and by the Kinoshita-Lee-Nauenberg theorem [5] (see also [1]). In turn, for the calculation of the functions $f_{n}\left(E_{e}\right)$ and $h_{n}^{(\ell)}\left(E_{e}\right)$, where $\ell=1,2[2]$ and $\ell=3,4$ [see Eq. (A8)], one may use both the Lorentz covariant FPM regularization with an infinitesimal photon mass $\mu$ and the infrared cutoff $\omega_{\min }$ regularization. Indeed, the contributions of the integral $J\left(\beta, \kappa_{\mathrm{IR}}\right)$, the regularization of which depends on the regularization procedure [see Eqs. (A10) and (A12)], cancel themselves in the differences $\lim _{\kappa_{\mathrm{IR}} \rightarrow 0}\left[g_{\beta_{c}^{-} \gamma}^{(i)}\left(E_{e}, \kappa_{\mathrm{IR}}\right)-g_{\beta_{c} \gamma}^{(1)}\left(E_{e}, \kappa_{\mathrm{IR}}\right)\right]$, where $i=2,3,4,5,6$, and the results do not depend on the regularization procedure.
[1] A. N. Ivanov, M. Pitschmann, and N. I. Troitskaya, Neutron beta decay as a laboratory for testing the standard model, Phys. Rev. D 88, 073002 (2013).

[2] A. N. Ivanov, R. Höllwieser, N. I. Troitskaya, M. Wellenzohn, and Ya. A. Berdnikov, Precision analysis of electron energy spectrum and angular distribution of neutron beta decay with polarized neutron and electron, Phys. Rev. C 95, 055502 (2017).

[3] A. N. Ivanov, R. Höllwieser, N. I. Troitskaya, M. Wellenzohn, and Ya. A. Berdnikov, Tests of the Standard 
Model in neutron beta decay with polarized neutron and electron, and unpolarized proton, Phys. Rev. C 98, 035503 (2018).

[4] M. Tanabashi et al. (Particle Data Group), Review of particle physics, Phys. Rev. D 98, 030001 (2018).

[5] A. Sirlin, General properties of the electromagnetic corrections to the beta decay of a physical nucleon, Phys. Rev. 164, 1767 (1967).

[6] R. T. Shann, Electromagnetic effects in the decay of polarized neutrons, Nuovo Cimento A 5, 591 (1971).

[7] V. Gudkov, G. I. Greene, and J. R. Calarco, General classification and analysis of neutron beta-decay experiments, Phys. Rev. C 73, 035501 (2006); V. Gudkov, Asymmetry of recoil protons in neutron beta decay, Phys. Rev. C 77, 045502 (2008).

[8] D. H. Wilkinson, Analysis of neutron beta decay, Nucl. Phys. A377, 474 (1982).

[9] T. D. Lee and C. N. Yang, Question of parity conservation in weak interactions, Phys. Rev. 104, 254 (1956).

[10] T. D. Lee, R. Oehme, and C. N. Yang, Remarks on possible noninvariance under time reversal and charge conjugation, Phys. Rev. 106, 340 (1957).

[11] J. D. Jackson, S. B. Treiman, and H. W. Wyld, Jr., Possible tests of time reversal invariance in beta decay, Phys. Rev. 106, 517 (1957).

[12] M. E. Ebel and G. Feldman, Further remarks on Coulomb corrections in allowed beta transitions, Nucl. Phys. 4, 213 (1957).

[13] P. Herczeg, Beta decay beyond the standard model, Prog. Part. Nucl. Phys. 46, 413 (2001).

[14] N. Severijns, M. Beck, and O. Naviliat-Cuncic, Tests of the standard electroweak model in beta decay, Rev. Mod. Phys. 78, 991 (2006).

[15] V. Cirigliano, J. Jenkins, and M. González-Alonso, Semileptonic decays of light quarks beyond the standard model, Nucl. Phys. B830, 95 (2010).

[16] T. Bhattacharya, V. Cirigliano, S. D. Cohen, A. Filipuzzi, M. González-Alonso, M. L. Graesser, R. Gupta, and H.-W. Lin, Probing novel scalar and tensor interactions from (ultra)cold neutrons to the LHC, Phys. Rev. D 85, 054512 (2012).

[17] V. Cirigliano, M. Gonzáles-Alonso, and M. L. Graesser, Non-standard charged current interactions: Beta decays versus the LHC, J. High Energy Phys. 02 (2013) 046.

[18] V. Cirigliano, S. Gardner, and B. Holstein, Beta decays and non-standard interactions in the LHC era, Prog. Part. Nucl. Phys. 71, 93 (2013).

[19] S. Gardner and C. Zhang, Sharpening Low-Energy, Standard-Model Tests via Correlation Coefficients in Neutron Beta Decay, Phys. Rev. Lett. 86, 5666 (2001).

[20] S. Gardner and B. Plaster, Framework for maximum likelihood analysis of neutron beta decay observables to resolve the limits of the V-A law, Phys. Rev. C 87, 065504 (2013).

[21] H. Abele, Precision experiments with cold and ultra-cold neutrons, Hyperfine Interact. 237, 155 (2016).

[22] A. N. Ivanov, R. Höllwieser, N. I. Troitskaya, M. Wellenzohn, and Ya. A. Berdnikov, Precision theoretical analysis of neutron radiative beta decay to order $O\left(\alpha^{2} / \pi^{2}\right)$, Phys. Rev. D 95, 113006 (2017).

[23] H. Abele, The neutron. Its properties and basic interactions, Prog. Part. Nucl. Phys. 60, 1 (2008).
[24] D. Mund, B. Märkisch, M. Deissenroth, J. Krempel, M. Schumann, H. Abele, A. Petoukhov, and T. Soldner, Determination of the Weak Axial Vector Coupling from a Measurement of the Beta-Asymmetry Parameter A in Neutron Beta Decay, Phys. Rev. Lett. 110, 172502 (2013).

[25] B. Märkisch, H. Mest, H. Saul, X. Wang, H. Abele, D. Dubbers, M. Klopf, A. Petoukhov, C. Roick, T. Soldner, and D. Werder, Measurement of the weak axial-vector coupling constant in the decay of free neutrons using a pulsed cold neutron beam, arXiv:1812.04666.

[26] A. Czarnecki, W. J. Marciano, and A. Sirlin, The Neutron Lifetime and Axial Coupling Constant Connection, Phys. Rev. Lett. 120, 202002 (2018).

[27] J. M. Blatt and V.F. Weisskopf, Theoretical Nuclear Physics (John Wiley \& Sons, New York, 1952).

[28] J. D. Jackson, S. B. Treiman, and H. W. Wyld, Jr., Coulomb corrections in allowed beta transitions, Nucl. Phys. 4, 206 (1957); Note on relativistic coulomb wave functions, $\mathrm{Z}$. Phys. 150, 640 (1958).

[29] E. K. Konopinski, in The Theory of Beta Radioactivity (Clarendon Press, Oxford, 1966).

[30] R. Pohl et al., The size of the proton, Nature (London) 466 , 213 (2010).

[31] A. N. Ivanov, R. Höllwieser, N. I. Troitskaya, and M. Wellenzohn, Proton recoil energy and angular distribution of neutron radiative beta decay, Phys. Rev. D 88, 065026 (2013).

[32] C. Itzykson and J.-B. Zuber, in Quantum Field Theory (McGraw-Hill Inc., New York, 1980).

[33] S. Weinberg, in The Quantum Theory of Fields, Foundations (Cambridge University Press, New York, 1995), Vol. 1, p. 472.

[34] N. N. Bogoliubov and D. V. Shirkov, in Introduction to the Theory of Quantum Fields (Interscience Publishers Inc., New York, 1959).

[35] A. Czarnecki, W. J. Marciano, and A. Sirlin, Precision measurements and CKM unitarity, Phys. Rev. D 70, 093006 (2004).

[36] W. J. Marciano and A. Sirlin, Radiative Corrections to $\beta$ Decay and the Possibility of a Fourth Generation, Phys. Rev. Lett. 56, 22 (1986).

[37] W. J. Marciano and A. Sirlin, Improved Calculation of Electroweak Radiative Corrections and the Value of $V($ ud), Phys. Rev. Lett. 96, 032002 (2006).

[38] A. N. Ivanov, R. Höllwieser, M. Wellenzohn, N. I. Troitskaya, and Ya. A. Berdnikov, Internal bremsstrahlung of beta decay of atomic ${ }_{16}^{35} \mathrm{~S}$, Phys. Rev. C 90, 064608 (2014).

[39] T.D. Lee and C.N. Yang, Charge conjugation, a new quantum number $G$, and selection rules concerning a nucleon anti-nucleon system, Nuovo Cimento 3, 749 (1956).

[40] S. Weinberg, Charge symmetry of weak interactions, Phys. Rev. 112, 1375 (1958).

[41] A. N. Ivanov, Lorentz structure of vector part of matrix elements of transitions $n \leftrightarrow p$, caused by strong lowenergy interactions and hypothesis of conservation of charged vector current, J. Phys. G 45, 025004 (2018).

[42] J. C. Hardy and I. S. Towner, Superallowed $0^{+} \rightarrow 0^{+}$ nuclear beta decays: 2014 critical survey, with precise 
results for $V_{u d}$ and CKM unitarity, Phys. Rev. C 91, 025501 (2015).

[43] M. González-Alonso, O. Naviliat-Cuncic, and N. Severijns, New physics searches in nuclear and neutron beta decay, Prog. Part. Nucl. Phys. 104, 165 (2019).

[44] A. N. Ivanov, Comment on "On the implementation of CVC in weak charged-current proton-neutron transitions" by C. Giunti, arXiv:1602.00215; arXiv:1705.09573.

[45] S. Weinberg, Role of strong interactions in decay processes, Phys. Rev. 106, 1301 (1957).

[46] F. Glück, Order- $\alpha$ radiative correction to ${ }^{6} \mathrm{He}$ and ${ }^{32} \mathrm{Ar} \beta$ decay recoil spectra, Nucl. Phys. A628, 493 (1998).
[47] K. Mitchel, Tables of the function $\int_{0}^{z} \frac{-\log |1-y|}{y} d y$, with an account of some properties of this and related functions, Philos. Mag. J. Sci. 40, 351 (1949).

[48] L. Lewin, in Polylogarithms and Associated Functions (North-Holland, Amsterdam, 1981).

[49] J. S. Nico et al., Observation of the radiative decay mode of the free neutron, Nature (London) 444, 1059 (2006).

[50] R. L. Cooper et al., Radiative beta decay of the free neutron, Phys. Rev. C 81, 035503 (2010).

[51] M. J. Bales et al. (RDK II Collaboration), Precision Measurement of the Radiative Beta Decay of the Free Neutron, Phys. Rev. Lett. 116, 242501 (2016). 